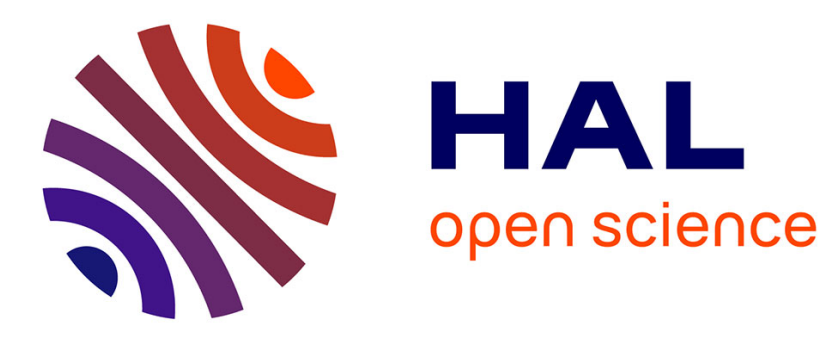

\title{
Standalone Extraction of Tonal Components from Aeroacoustic Signals
}

Jérôme Antoni, Quentin Leclère, Alice Dinsenmeyer, Emmanuel Julliard, Simon Bouley, Christophe Picard, Pieter Sijtsma

\section{To cite this version:}

Jérôme Antoni, Quentin Leclère, Alice Dinsenmeyer, Emmanuel Julliard, Simon Bouley, et al.. Standalone Extraction of Tonal Components from Aeroacoustic Signals. AIAA Journal, 2021, pp.1-16. 10.2514/1.J060288 . hal-03352595

\section{HAL Id: hal-03352595 \\ https://hal.science/hal-03352595}

Submitted on 23 Sep 2021

HAL is a multi-disciplinary open access archive for the deposit and dissemination of scientific research documents, whether they are published or not. The documents may come from teaching and research institutions in France or abroad, or from public or private research centers.
L'archive ouverte pluridisciplinaire HAL, est destinée au dépôt et à la diffusion de documents scientifiques de niveau recherche, publiés ou non, émanant des établissements d'enseignement et de recherche français ou étrangers, des laboratoires publics ou privés. 


\title{
Standalone Extraction of Tonal Components from Aeroacoustic Signals
}

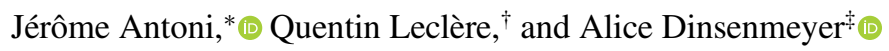 \\ University of Lyon, INSA Lyon, 69621 Villeurbanne, France \\ Emmanuel Julliard $\stackrel{\S}{-}$ \\ Airbus Operations S.A.S., 31300 Toulouse, France \\ Simon BouleyI and Christophe Picard** \\ MicrodB, F-69134 Ecully, France \\ and \\ Pieter Sijtsma立 \\ PSA3, 9493 TE De Punt, The Netherlands \\ https://doi.org/10.2514/1.J060288
}

\begin{abstract}
Aeroacoustic signals, as typically returned by recordings of pressure fluctuations generated by rotating machines, often exhibit rich mixtures of tonal and broadband components. The separate analysis of these constituents is important from an engineering point of view, as they relate to different physical mechanisms. This paper is concerned with the extraction of tonal components. The characteristics of aeroacoustic signals can make this task challenging, because tones are numerous, nonharmonically related, and subjected to random modulations. A solution is proposed based on the theory of angle-time cyclostationarity, which seems flexible enough to deal with these constraints. A special effort is made to render the methodology as standalone as possible. This is achieved by automatically setting up the leading parameters with data-driven strategies. The methodology is illustrated on counter-rotating open rotor data that are known to be challenging.
\end{abstract}

\section{Nomenclature}

$\mathcal{A}=$ set of tone frequencies

$C(\gamma)=$ cost function for selecting kernel bandwidth $\gamma$

$c_{k}(t)=$ time-varying Fourier coefficient

$c_{k i}=$ short-time Fourier coefficient

$e(t)=$ residual signal after removal of estimated tonal part

$\mathcal{F}=$ robust smoothing filter

$f \quad=$ frequency, $\mathrm{Hz}$

$I=$ number of time instants in short-time angular Fourier series

$j \quad=$ imaginary unit

$L \quad=\quad$ signal length (samples)

$M \quad=$ number of sensors

$N_{w} \quad=\quad$ kernel length (samples)

$p(t)=$ tonal part of aeroacoustic signal

$S_{\mathrm{BL}}=$ spectral baseline of signal $y$

$S_{y}(f)=$ power spectral density of signal $y$

$t=$ time index

$\boldsymbol{v}_{k}=k$ th eigenvector

$w(t)=$ kernel of short-time (angular) Fourier transform

$x(t)=$ broadband part of aeroacoustic signal

$\boldsymbol{Y}(f)=$ matrix of short-time Fourier coefficients of signals $y_{m}(t), m$ is equal to $1, \ldots, M$ at frequency $f$

$y(t)=$ measured aeroacoustic signal

$\alpha=$ tone order (counts per revolution)

$\Delta=$ time shift in short-time angular Fourier series

Received 30 October 2020; revision received 1 July 2021; accepted for publication 8 July 2021; published online 20 September 2021. Copyright (C) 2021 by the American Institute of Aeronautics and Astronautics, Inc. All rights reserved. All requests for copying and permission to reprint should be submitted to CCC at www.copyright.com; employ the eISSN 1533-385X to initiate your request. See also AIAA Rights and Permissions www.aiaa.org/randp.

*Professor, Laboratoire Vibrations Acoustique (LVA), EA677; jerome .antoni@insa-lyon.fr.

${ }^{\dagger}$ Assistant Professor, Laboratoire Vibrations Acoustique (LVA), EA677.

${ }^{*}$ Research Engineer, Laboratoire Vibrations Acoustique (LVA), EA677.

${ }^{\S}$ Acoustic Engineer, Acoustics Department.

${ }^{\top} \mathrm{Ph} . \mathrm{D} .$, Acoustic Engineer.

***Deputy Director.

${ }^{\dagger}$ Director.

$\begin{array}{lll}\gamma & = & \text { spectral bandwidth of kernel } \\ \lambda_{k} & = & k \text { th eigenvalue } \\ \Theta & = & \text { angular period, } \text { rad } \\ \theta & = & \text { reference angle, rad } \\ \chi^{2} & = & \text { chi-squared distribution }\end{array}$

Subscripts

$i=$ time index in short-time angular Fourier series

$k=$ order index in short-time angular Fourier series

$m \quad=$ sensor number

\section{Introduction}

EROACOUSTIC signals, as typically returned by recordings of $A$ pressure fluctuations generated by rotating machines, often have a complex structure. They show combination of tonal components, with deterministic characteristics, and broadband components, which are fundamentally random [1]. The separate analysis of the two types of components is of importance for the experimenter as they carry different elements of information [2].

For example, in the case of a turbomachine-which is the application that originally motivated the present work-tonal components are produced by various mechanisms linked to the rotation of the fan or propeller. Tones are seen as deterministic because their frequencies and, to some extent, their magnitudes, can be determined a priori from first principles of physics. In the most typical case, the dominant tones are located at the blade-passing frequency (BPF) and its harmonics. The BPF and its harmonics are themselves likely to be surrounded by sidebands of the rotor frequency. Even in this configuration, the resulting line spectrum may evidence several tens or hundreds of harmonics in the audible range. More complex configurations exist, for instance, when two or more rotors interact. The counter-rotating open rotor (CROR) is one such example, which is analyzed in Sec. VI of this paper.

Contrary to tones, broadband components are characterized by continuous power spectral densities (PSDs). They are modeled as random processes, with persistent (i.e., with infinite support) but unpredictable (i.e., nondeterministic) trajectories in time. This random nature is also reflected by the difficulty to construct physical models. Broadband components are due to numerous phenomena, 
which can be roughly divided into those linked to interactions with the external medium and those involving interaction with the rotation of the propeller. The first instance typically includes turbulent flows and external sources that globally participate to background noise. The corresponding random processes can be modeled as stationary. The second instance includes all phenomena due to the rotation of the blades, other than those leading to tones. The corresponding random processes are thus likely to be modulated by the BPF and, therefore, are better modeled as cyclostationary, i.e., with periodic statistics (see [3] for an early introduction to cyclostationary processes, [4] for a general introduction to some of their applications in vibrations and acoustics, and [5] for one of the first applications in aeroacoustics).

A third type of component, namely, narrowband random components, may exist in aeroacoustic signals. Narrowband random components may either originate from a tone that undergoes random fluctuation, or from a broadband random source that passes through a sharp bandpass filter. As such, they illustrate a continuous transition from tonal to broadband components and they are often assigned to one of these two categories.

Because tonal and broadband components correspond to different noise generating mechanisms, it is useful to analyze them separately. The present work proposes solutions to achieve this separation in experimental data, as returned by an array of microphones.

The model adopted throughout the document is

$$
y_{m}(t)=p_{m}(t)+x_{m}(t), \quad m=1, \ldots, M
$$

where $y_{m}(t)$ stands for the pressure signal measured by the $m$ th sensor in an array of $M$ elements, $p_{m}(t)$ for its tonal part, and $x_{m}(t)$ for its broadband part. The aim is to extract the tonal part $p_{m}(t)$ from the simultaneous measurements $y_{m}(t), m=1, \ldots, M$, on each sensor $m$. As a byproduct, the broadband part $x_{m}(t)$ is obtained by subtraction.

The issue is not as easy as it first appears. Because a tonal component is modeled by a phasor that rotates synchronously with the rotor, it is periodic with respect to an angle of rotation. Aeroacoustic signals being sampled in time, periodicity is preserved if and only if the rotor speed is perfectly constant. This is rarely the case in practice, even when the measurements are taken under steady conditions. Experimental signals show that, even under controlled configurations, speed variations of at least a few per mille are commonplace, which is yet noticeable on long records. As a consequence, tones do not produce exact lines in the spectrum, but tend to be spread in frequency. This phenomenon can be easily fixed by resampling the signal in the angular domain, i.e., changing time, as the descriptive variable of the signals, for the angle of rotation of the rotor. This practice is, for instance, commonplace in machine health monitoring [6]. One difficulty arises, however, when the signals contain families of tones related to different and independentincommensurable - rotation frequencies (e.g., as with the CROR), because there is no common angular variable that allows simultaneous resynchronization of all components. Another issue is that, even under perfectly constant speed, the acoustical wave fronts get distorted by random fluctuations in the medium of propagation (convection, chopping by blade rotation, etc.). It can be shown that this effect is similar to clock noise, for which a first model is additive Brownian noise in the phase. The corresponding shape of the tones is then a Lorentzian distribution in the frequency spectrum, the extent of which is unbounded (the Lorentzian distribution decreases asymptotically like the inverse of the square frequency around the central frequency). This kind of fluctuation cannot be fixed with angular resampling.

The problem of extracting a set of tones with known frequencies from additive broadband noise has been approached from several angles in the literature. The simplest technique consists in clipping or erasing the peaks in the Fourier spectrum of the signal. This approach only applies to tonal components that protrude from the spectrum, i.e., whose power is greater than the power of surrounding noise- $-a$ situation that awkwardly depends on the frequency resolution set by the user.限 In practice, this condition is rarely met for higher-order

蛏 $A$ denotes the amplitude of the tone, $\sigma^{2}$ the power spectral density in its vicinity, and $\Delta f$ the user-defined frequency resolution, then the requirement for a tone to be detected and thus possibly clipped is $A^{2} \geq 2 \sigma^{2} \Delta f$. harmonics, which rapidly decrease below the noise level. Another drawback of the peak clipping technique is that the tonal signal can hardly be reconstructed in the time domain. Another popular approach is the time-synchronous average, which consists in averaging periods of the signal $[7,8]$. If correctly synchronized to the true period, the average is therefore constructive for the underlying periodic components and destructive for random (or other asynchronous) components. When processing discrete-time signals, the synchronous average requires the signal to be coded with an integer number of samples per period. In practice, this is forced by resampling in the angular domain. Under these conditions, the synchronous average can be shown to be optimal in the least square sense; i.e., it is the filter that minimizes the sum of the squared difference with the actual periodic signal. Generalizations of the synchronous average that accept slow modulations, as often encountered with rotating machines, have been proposed in [9,10]. A rather similar method has been independently proposed in [1] , with the additional advantage of being free of a tachymetric signal. The main drawback of the synchronous average and its extensions is the requirement of resampling (or resynchronizing) in the angular domain as many times as there are incommensurable periods in the signal. As demonstrated in Sec. VI, this makes the synchronous average impracticable on the $\mathrm{CRO} \overline{\mathrm{R}}$ data that involve numerous tonal interactions at incommensurable frequencies. An almost equivalent formulation of the synchronous average is by means of a Fourier series (equivalence is exact when the measured signal contains an integer number of periods), yet without the aforementioned restriction [12]. The tonal components are then reconstructed from their estimated Fourier coefficients on an arbitrary set of frequencies [13]. Besides, this may be advantageous when only a limited number of tonal components are of interest. The frequency domain interpretation of the synchronous average is a frequency gain in the form of a Dirichlet kernel, i.e., with equispaced peaks that extracts the harmonics of the periodic signal [14]. More generally, tonal components can be directly removed from the frequency domain by designing a combfilter that sharply encloses the harmonics with unit gain and zeroes all other components. This can be easily generalized to quasi-periodic signals, i.e., signals that are not periodic but that are still described by a Fourier series (e.g., which contains tones with incommensurate frequencies). Combfilters are quite flexible because they can be implemented in various ways [15]. They also generalize most of the other techniques dedicated to tone extraction. Another popular class of methods is based on the use of references to design conditioned filters [16]. The references must be perfectly correlated with the signal to be extracted, as ideally returned by a linearly filtered version; this implies that no harmonic is missing. Unfortunately, when the reference is provided by a tachymetric signal, there is no guarantee that this condition is actually met. The estimation of the conditioned filter also requires the signals to be stationary, which ideally requires resampling in the angular domain. An adaptive estimation of the filter is surely possible, yet this is at the detriment of the estimation performance and of the frequency resolution. If the tonal components were perfectly stable in time (constant rotor speed and absence of clock noise $\underline{\S \S}$ ), they could be easily extracted by one of the aforementioned methods. As previously discussed, this ideal situation is not necessarily met in practice. In that context, many authors have designed tracking algorithms that are apt to extract tonal components with slow time variations in their amplitude and phase. Tracking algorithms can be seen as generalizations of the combfilters [17] and conditioned filters that adapt themselves to nonstationary evolutions of the signal. The Kalman filter $[18,19]$, the RLS filter, the Vold-Kalman filter [20,21], etc., are popular examples. They pertain to model-based methods, where the dynamics of the tones is explicitly modeled. Although modelbased methods are theoretically able to return extremely accurate results, the main drawback encountered by several of them is to be limited in the number of tones that they can extract simultaneously (technically, they involve the solution of a system of equations with dimension equal to the number of tones to track, and this at each time step). The Vold-Kalman stands as a particular case as it can be

\footnotetext{
${ }^{\S}$ Clock noise refers to random fluctuations of the tone frequency.
} 
applied sequentially to each tone (or subset of tones), as demonstrated in [21-23], a practice that might be otherwise hazardous with those model-based methods that explicitly assume the residual signal to be a stationary white noise (see, e.g., [24]). Even when tracking tones sequentially, the Vold-Kalman filter mathematically involves an algebraic system of equations of dimension equal to the signal length. Its sparse banded structure is, however, exploited in an efficient implementation. A shown in Sec. II.C, the complexity of the method introduced in this paper is comparable to that of the Vold-Kalman filter. Therefore it offers a valid alternative. First, because it solves the same problem from a different approach, yet with similar performance. This is valuable from both a theoretical and a practical point of view. Second, because it is easily amenable to automated processing, as shown in Sec. III. Third, because it is easily combined with spatial filtering when multiple sensors are available, as demonstrated in Sec. IV.

To summarize, the extraction of tones from aeronautics measurements is faced with four constraints. First, the signals are periodic in angle although recorded in time. Second, they contain clock noise. Third, the number of tonal components may be large (note that the case of coincident or crossing orders is not of concern in this paper). Fourth, they have a finite duration. These four constraints constitute the main challenges considered in this work. None of the techniques listed in the previous paragraph can easily deal with them altogether.

The present paper introduces an approach rooted in the theory of angle-time cyclostationary (AT-CS) processes, which specifically accounts for the particularity of aeroacoustic signals. It elaborates on the material first presented in Ref. [25].

The first challenge is solved by introducing an angle-time formulation of the Fourier series, which involves time-varying Fourier coefficients paired with angle-varying phasors synchronized to the rotor speed $[26,27]$. The advantages of this formulation are that 1$)$ it does not need to resample the signal in the angular domain (an operation that always introduces some errors in the form of spectral alias) and 2) it can easily accommodate synchronization to several incommensurable frequencies.

The second constraint relates to the fact that the tones undergo uncontrolled random modulations of their amplitude and phase. By "uncontrolled," it is meant that they cannot be synchronized to any measured rotor speed in the system. A short-time version of the angular Fourier series is proposed that leads to time-varying Fourier coefficients apt to track slow (as compared with the tone frequency) phase and amplitude modulations. Conditions for the invertibility of the short-time angular Fourier series (STAFS) are also established.

The third constraint implies that the signal-to-noise ratio (SNR, defined as the ratio of the tone power to the noise PSD evaluated at the tone frequency) is upper bounded. Consequently, only tones that emerge from background noise can be reasonably estimated, the other ones being statistically indistinguishable from noise. This becomes an issue when the number of candidate tones to be removed is large - the fourth constraint-because it involves unnecessary computations of tones that are below the critical SNR, but also because it involves numerous Fourier coefficients whose values are dominated by estimation errors. The signal reconstructed from the Fourier series based on these Fourier coefficients thus accumulates estimation errors (whose energy is proportional to the number of Fourier coefficients) to a point that may become unacceptable. This is solved by setting up a test of hypothesis, which keeps only the Fourier coefficients that are statistically significant and implicitly zeroes all other coefficients.

Another aim of the present work is to make the above processing as autonomous as possible. This is already partly achieved thanks to the aforementioned test of hypothesis that automatically selects the significant Fourier coefficients in a list of candidates. Another crucial step that has to be automated is the selection of the kernel bandwidth used in the STAFS. A statistical test is designed for this purpose, which consists in selecting the optimal bandwidth as the one that achieves the closest fit between the PSD of the residual signal (after extraction of the tones in the time domain by using the aforementioned method) and the spectral baseline (estimated by robust smoothing the PSD of the original signal). Special attention has been given to make the test reasonably fast in order to keep it realistic for practical purposes.

The proposed methodology is able to extract common tones from a set of signals measured in an array of microphones $(M>1$ in model 1). Although this configuration could be handled by processing each signal independently, it would not take advantage of the fact that the same tones are likely to be shared by several signals. The SNR of the extracted tones can be potentially increased by a proportion equal to the number of sensors in the array. One way to deal with this scenario is to apply the above algorithm on the eigenspectra of the signals-i.e., the eigenvalues displayed as functions of order of the cross-spectral matrix. The latter is the correlation matrix of the short-time Fourier coefficients of the signals, calculated for each order of interest. The tonal components of each signal can then be reconstructed by projecting the retained coefficients on the signal subspace.

This paper is organized as follows. Section II first introduces a description of the statistical properties of the signals within the framework and AT-CS processes. It then proposes a solution to the extraction of tones based on the STAFS. For simplicity, the solution is first given in the single sensor case $(M=1)$. Next, Sec. III investigates how to make the extraction of tones standalone through automatic setting of parameters. Section IV extends the method to multiple simultaneous measurements $(M \overline{>} 1)$. Section $\underline{\mathrm{V}}$ validates the method on synthetic data. Finally, Sec. VI provides an experimental validation by illustrating the method on CROR data, which introduce several practical challenges.

The following notations are used throughout the documents. Bolditalic lower case letters are used to denote vectors. Bold upper case letters are used to denote matrices. The imaginary number is noted $j$. $\|\boldsymbol{a}\|$ stands for the $\ell_{2}$ norm of a vector and $\|\boldsymbol{A}\|$ for the Frobenius of a matrix $\boldsymbol{A} . \boldsymbol{A}^{H}$ is the Hermitian transpose-i.e., conjugate transposeof matrix $\boldsymbol{A}$. In general, the symbol ${ }^{\wedge}$ over a quantity will indicate that this quantity is estimated; e.g., $\hat{\theta}$ is an estimate of $\theta$.

\section{Problem Statement}

This section introduces the principles that are used to achieve the extraction of tonal components. The short-time angular Fourier series (STAFS) is then introduced as the basic tool for extracting and resynthesizing tonal components.

\section{A. Signal Model}

The signal model is first resumed in the case of one measurement, as returned by a single sensor. The extension to multiple simultaneous measurements will be addressed in Sec. IV. As stated in model (1), let $y(t)$ denote the measured time record, at discrete time instants indexed by $t=0,1,2, \ldots, L-1$. The measurements comprise the superposition of a tonal part $p(t)$ and a broadband part $x(t)$, such that

$$
y(t)=p(t)+x(t)
$$

These two parts are characterized by different statistical properties, hereafter described in assumptions $\mathrm{H} 1$ and $\mathrm{H} 2$.

H1) $p(t)$ embodies all the tonal components, which are listed by a set of frequencies $\mathcal{A}$. Each tonal component in the list is a sinusoidal function of the reference angle $\theta$, for instance, the angle of rotation of a rotor, propeller, or fan. It is therefore, strictly speaking, an angleperiodic function. In addition, a tonal component may undergo slow and unpredictable modulations that are functions of time, which make it quasi-angle-periodic, i.e., seemingly angle-periodic on a short-time basis, but random on the long-term. Such a process is referred to as AT-CS. It reads

$$
p(t)=\sum_{k \in \mathcal{A}} c_{k}(t) e^{j \alpha_{k} \theta(t)}, \quad j^{2}=-1
$$

where $\alpha_{k}$ stands for the order of the $k$ th tone. Note that the orders are allowed to take arbitrary values and, in particular, they are not constrained to be harmonically related. The time-dependent Fourier coefficients $c_{k}(t)$ are assumed stationary and narrow band. 
The later assumption is obviously required for the model to remain identifiable.

The model can be easily extended to accept sets of tonal components with different reference angles (for instance, in the case of the CROR engine with rear and front rotors; see Sec. VI). It then takes the general form

$$
p(t)=\sum_{l \in \mathcal{B}} p_{l}(t)=\sum_{l \in \mathcal{B}} \sum_{k \in \mathcal{A}_{l}} c_{l k}(t) e^{j \alpha_{l k} \theta_{l}(t)}
$$

with $\left\{\theta_{l}(t)\right\}$ a set of angles of rotation that are not synchronously related; i.e., there does not exist any constant $\kappa_{l m}$ such that $\theta_{l}(t) / \theta_{m}$ $(t)=\kappa_{l m}, \forall(l, m) \in \mathcal{B}^{2}$.

$\mathrm{H} 2) x(t)$ is broadband random, i.e., with a "continuous" PSD and bandwidth much larger than that of the time-dependent Fourier coefficients $c_{k}(t)$. So far, no other assumption is necessary concerning its probability density function (PDF), except some mild conditions for the short-time Fourier coefficients to tend to Gaussianity. In particular, it is allowed to be purely AT-CS on the second-order (AT-CS2), i.e., zero-mean (in the ensemble average sense) and nonstationary with angle-periodic statistics [28].

\section{B. Solution Based on the Short-Time Angular Fourier Series}

This subsection explains how to estimate the time-varying Fourier coefficients $c_{k}(t)$ of Eq. (3) from the measured signal $y(t)$ and, subsequently, how to reconstruct the tonal part $p(t)$.

\section{Principle of the Extraction}

Based on assumptions $\mathrm{H} 1$ and $\mathrm{H} 2$, the signal model embodied by Eqs. (2) and (3) or its generalization (4) provides a complete description of tonal components immersed in background noise. Therefore, given the reference angle $\theta$, the extraction of the tones is fully solved by identifying the time-varying coefficients $c_{k}(t)$ assigned to each phasor in the decomposition. The feasibility of the approach relies on two questions: Can the time-varying Fourier coefficients be uniquely identified? If so, how to identify them? The first question is conceptual and relates to the identifiability of the model. The other one is technical and relates to methodology.

Identifiability is guaranteed if the angle-time Fourier series (2) uniquely defines the tonal part on the one hand, and cannot model part of the broadband part on the other hand. The first condition is satisfied if the bandwidths of the time-varying coefficients are narrow enough. At nearly constant rotation speed, this means that they should be smaller than the spacing between the tones. The second condition is more difficult to satisfy because, as soon as the Fourier coefficients are made time-varying, they are apt to capture part of the broadband noise. However, if the time variations are slow enough — which again means narrow bandwidths - this effect can be neglected.

Assuming that the model is identifiable, the next question concerns the estimation of the time-varying Fourier coefficients. Although least square estimation is conceivable $[20,21,29]$, it would rapidly become intractable as the number of tones becomes large. Besides, the classical Fourier transform cannot be used because the Fourier coefficients are functions of time, whereas the phasors are functions of angle. A solution based on the STAFS, a generalization of the Fourier series to AT-CS processes, is introduced hereafter.

\section{Time-Varying Fourier Coefficients}

The estimation of the Fourier coefficients $c_{k}(t)$ has to take two specificities into account. The first one is that they relate to angleperiodic phasors $e^{j \alpha_{k} \theta(t)}$, which means that Fourier integrals have to be calculated with respect to angle. Because the position of the present work is to keep all processings in the time domain in order to avoid cumbersome resampling in the angular domain, the angle $\theta(t)$ has to be considered as an explicit function of time. The second specificity is to compute the Fourier coefficients on a short-time basis in order to track their time variations. Therefore, an estimate of $c_{k}(t)$, the Fourier coefficient of the $k$ th harmonic at time datum $i \Delta, \Delta \in \mathbb{Z}^{+}$, $i=0, \ldots, I-1$, is

$$
\begin{aligned}
c_{k i} & =\frac{1}{\phi_{i}} \sum_{t \in \mathbb{Z}} y(t) e^{-j \alpha_{k} \theta(t)} w(\gamma(t-i \Delta)) \dot{\theta}(t), \\
\phi_{i} & =\sum_{t \in \mathbb{Z}} w(\gamma(t-i \Delta)) \dot{\theta}(t)
\end{aligned}
$$

where $w(\gamma t)$ is a smooth, nonnegative, and symmetric kernel (symmetry is assumed for simplicity, although not strictly necessary) with finite support and spectral bandwidth $\gamma$. It is noteworthy that the summation in Eq. (5) is actually finite, on the interval $t=i \Delta, \ldots, i \Delta$ $+N_{w}-1$, with $N_{w}$ the kernel length, which is a function of $\gamma$ (for instance, with a Hann window $w(t)=(1 / 2)(1-\cos (2 \pi \gamma t)$ ), $\left.N_{w}=1 / \gamma\right)$. For a record $y(t)$ of length $L$, a window length $N_{w}$, and a stride $\Delta$, there are

$$
I=\left\lfloor\left(L-\left(N_{w}-\Delta\right)\right) / \Delta\right\rfloor
$$

time instants where to compute the Fourier coefficients, hereafter denoted as snapshots.

Besides, the following assumption (H3) will be made from now on:

H3) In order for the instantaneous Fourier coefficients $c_{k i}$ to properly track the time variations of $c_{k}(t)$, the spectral bandwidth $\gamma$ of kernel $w(t)$ is broader than the bandwidth of $c_{k}(t)$.

Under this condition,

$$
\hat{c}_{k}(t)=\kappa \sum_{i=0}^{I-1} c_{k i} w(\gamma(t-i \Delta))
$$

with $\kappa=1 /\left(\sum_{i \in \mathbb{Z}} w(\gamma i \Delta)\right)$ returns an estimate of $c_{k}(t)$ in the form of an interpolation based on kernel $w(\gamma t)$ (see Appendix).

\section{Synthesis of Tones}

Once the Fourier coefficients have been estimated, the tonal components can be extracted by using the reconstruction formula:

$$
\hat{p}(t)=\sum_{k \in \mathcal{A}} \hat{c}_{k}(t) e^{j \alpha_{k} \theta(t)}
$$

with $\hat{c}_{k}(t)$ as defined in Eq. (7). In the case of time-invariant Fourier coefficients, perfect reconstruction is guaranteed by the partitionof-unity condition:

$$
\sum_{i \in \mathbb{Z}} w(\gamma(t-i \Delta))=\mathrm{constant}
$$

Near-perfect reconstruction in the case of slowly varying Fourier coefficients is guaranteed by assumption $\mathrm{H} 3$ (see Appendix).

The above formula (8) can actually be used in several ways. One is to extract tones in a reduced list only (i.e., a subset of $\mathcal{A}$ ), for instance, tones related to the rear and front propellers, separately. Another possibility is to reconstruct the tonal components at a different speed than the measured ones, simply by adjusting the value of $\theta$. It is also possible to reconstruct unmodulated tones by replacing the Fourier coefficients $c_{k i}$ by equivalent but constant values, for instance, substituting $\left\|c_{k}\right\|=\left(\sum_{i=0}^{I-1}\left|c_{k i}\right|^{2} / I\right)^{1 / 2}$ for $\left|c_{k i}\right|$ (same power), thus leading to

$$
\hat{p}(t)=\sum_{k \in \mathcal{A}}\left\|\hat{c}_{k}\right\| e^{j \alpha_{k} \theta(t)}
$$

\section{Complexity}

The complexity of the proposed method is $L N_{w} / \Delta$ for both the computation of the short-time Fourier coefficients and for the synthesis of a tone. For instance, with a Hann window and 3/4 overlapwhich is the default choice used hereafter as it trivially satisfies 
condition (33) - the constant $N_{w} / \Delta=4$ remains insignificant as compared with $L$, so that complexity essentially scales with the signal length. In the general case of $K$ tones and $M$ channels, the complexity is $K M L$.

By comparison, the complexity of the Vold-Kalman filter is $L q^{2}$ for factorizing a sparse system of equations and $L q$ for the subsequent filtering of a tone, with $q$ the filter order (a typical choice is a two-pole filter, i.e., $q=2$ ). The factorization being computed only once (assuming a constant bandwidth), the complexity also scales as $K M L$ when several tones and channels are processed.

\section{Toward Automated Extraction of Tones}

The apparent simplicity of the proposed method hides some hitches. The first one is the selection of the spectral bandwidth $\gamma$ in Eq. (5). On the one hand, $\gamma$ is bounded downward by assumption H3; on the other hand, if too large, a large part of the noise $x(t)$ will be extracted together with the tonal components $p(t)-\mathrm{Eq}$. (A7) of Appendix establishes that the energy of this part is proportional to the noise PSD times the spectral bandwidth of the kernel. This is reminiscent of the identifiability issue discussed in Sec. II.B.1. The user is therefore faced with a difficult bias-variance tradeoff considering the fact that the bandwidth of the tones-according to which $\gamma$ is to be set-is a priori unknown. One possibility would be to estimate it from the width of the peaks in the spectrum, by visual inspection or some heuristic criterion (e.g., the $3 \mathrm{~dB}$ bandwidth). However, such an approach has been found fastidious due to the diversity of the spectral configurations. An automatic setting of the spectral bandwidth thus seems compulsory. This is the object of Sec. III.B.

The second hitch is again related to the fact that part of the noise $x(t)$ is unavoidably extracted together with the tones. When tones are below the level of noise, or simply when they are nil, the estimates are dominated by noise. This is clearly troublesome when it involves several components in the reconstruction formula (8) because the energy of noise then grows proportionally, up to a level that might become unacceptable. It is therefore necessary to identify those tones that are statistically nil-i.e., indistinguishable from noise for a given record length - and to remove them from the reconstruction. An automatic method to do so is proposed in Sec. III.C.

As will be seen later, the automatic selection of the spectral bandwidth and of the significant tones requires a good estimate of the noise PSD before the tones are extracted. This matter is treated as a preliminary step in the next subsection, where an original solution is proposed.

\section{A. Estimation of Spectral Baseline}

Based on assumptions $\mathrm{H} 1$ and $\mathrm{H} 2$, the noise PSD coincides with the spectral baseline of the measured signal $y(t)$, i.e., the line that passes smoothly through the broadband part of the spectrum as if peaks were absent. Hence, the idea is to estimate the PSD of $x(t)$ from the spectral baseline of $y(t)$. This is achieved by first estimating the PSD of signal $y(t)$ with a very fine frequency resolution, say, $S_{y}^{\text {fine }}(f)$, and next applying a robust smoothing filter, $\mathcal{F}$, with high resilience to peaks, to estimate the spectral baseline, $S_{\mathrm{BL}}=\mathcal{F}\left\{S_{y}^{\text {fine }}(f)\right\}$. For instance, a simple robust smoothing filter is offered by the running median, yet it may have a prohibitive computational cost when the number of spectral bins is large (as implied by a fine frequency resolution). A filter $\mathcal{F}$ with better computational efficiency is proposed herein. It consists of robustly fitting the logarithm $\ln S_{y}^{\text {fine }}(f)$ on a smooth spline basis (the rationale for taking the logarithm is to reduce the dynamics of the peaks and to get closer to a Gaussian distribution). The associated probabilistic model is

$$
\ln S_{y}^{\text {fine }}(f)=\underbrace{\sum_{n=1}^{N} b_{n} \phi_{n}(f)}_{\ln S_{\mathrm{BL}}(f)}+\epsilon(f)
$$

where $\left\{\phi_{n}(f) ; n=1, \ldots, N\right\}$ is a set of spline functions, $\boldsymbol{b}=$ $\left(b_{1}, \ldots, b_{N}\right)^{T}$ stands for a vector of coefficients, and $\epsilon(f)$ follows a leptokurtic PDF that embodies the occurrence of peaks in the spectrum. Assuming that the PDF of $\epsilon(f)$ is a generalized Gaussian with power $0<q<2$ (this choice is a matter of convenience, yet other possibilities are allowed, e.g., a $t$-Student, without much difference in the final results) and considering frequency bins $f_{j}$, $j=1, \ldots, F$, the maximum likelihood estimate of $\boldsymbol{b}$ is the solution of

$$
\hat{\boldsymbol{b}}=\underset{\boldsymbol{b}}{\operatorname{Argmin}} \sum_{j=1}^{F}\left\|\ln S_{y}^{\mathrm{fine}}\left(f_{j}\right)-\sum_{n=1}^{N} b_{n} \phi_{n}\left(f_{j}\right)\right\|^{q}
$$

which may be solved, for instance, with iterated reweighted least squares (IRLS). Finally, the spectral baseline is returned by

$$
S_{\mathrm{BL}}(f)=\exp \left(\sum_{n=1}^{N} \hat{b}_{n} \phi_{n}(f)\right)
$$

\section{B. Automatic Setting of Bandwidth}

The selection of the bandwidth $\gamma$ is a compromise between bias errors (amplified by too large values of $\gamma$ ) and stochastic errors (produced by too small values of $\gamma$ ). The two types of errors have different manifestations in the spectrum of the residual signal $e(t)=y(t)-\hat{p}(t)$ [an estimate of $x(t)$ in Eq. (2) ]. For $\gamma$ too small, the peaks of $p(t)$ are not well removed and parts of them are still present in the spectrum. For $\gamma$ too large, part of the noise is removed together with the peaks, which leaves large dips in the spectrum. The optimum is somewhere between these two extremes, where just enough of the peaks is removed so as to produce a smooth spectrum, without residual peaks or dips. The idea is to search for this compromise by making the PSD of $e(t)$ as close as possible to the spectral baseline $S_{\mathrm{BL}}(f)$.

To do so, the following short-time Fourier transform (STFT)

$$
\begin{aligned}
E_{i}\left(f_{k}\right) & =\frac{1}{\kappa^{\prime}} \sum_{t \in \mathbb{Z}} e(t) w\left(\gamma^{\prime}\left(t-i \Delta^{\prime}\right)\right) e^{-j 2 \pi t f_{k}}, \quad f_{k}=\frac{k}{N^{\prime}}, \\
k & =0, \ldots, N^{\prime}-1, \quad i=0, \ldots, I^{\prime}-1
\end{aligned}
$$

of the residual is considered, with $N^{\prime}$ the length of kernel $w(t)$ and $\kappa^{\prime}=\left(\sum_{t} w^{2}\left(\gamma^{\prime} t\right)\right)^{1 / 2}$. The differences with the Fourier coefficients of the STAFS in Eq. (5) must be highlighted. First, the STFT involves a transformation over the time variable and not angle. Second, it is normalized so that

$$
\hat{S}_{e}\left(f_{k}\right)=\frac{1}{I^{\prime}} \sum_{i=0}^{I^{\prime}-1}\left|E_{i}\left(f_{k}\right)\right|^{2}
$$

returns an estimate of the PSD of $e(t)$. Third, it does not have to be invertible, which places no constraint on the value of the stride $\Delta^{\prime}$. Fourth, the kernel shape might be different, although the same notation $w(t)$ is used here for simplicity. Fifth, the spectral bandwidth $\gamma^{\prime}$ is taken at least as small as the (assumed) lower bound of $\gamma$.

With these settings, it is proved in the Appendix that a spectral distance between $\hat{S}_{e}\left(f_{k}\right)$ and $S_{\mathrm{BL}}\left(f_{k}\right)$ in a band defined by indices $k \in \mathcal{K} \subset\left\{0, \ldots, N^{\prime} / 2\right\}$ is returned by

$$
C=\frac{1}{F} \sum_{k \in \mathcal{K}}\left(S_{k}-\ln S_{k}\right) \quad \text { where } S_{k}=\frac{I^{\prime} \cdot \hat{S}_{e}}{I_{\mathrm{eff}}^{\prime} \cdot S_{\mathrm{BL}}\left(f_{k}\right)}
$$

with $F$ the cardinal of $\mathcal{K}$ and $I_{\text {eff }}^{\prime}$ the effective number of snapshots as returned by Eq. (20). Clearly, this defines a cost function $C(\gamma)$ that depends on the spectral bandwidth $\gamma$ through the computation of the residual signal $e(t)$. Therefore, the optimal value of $\gamma$ is found as

$$
\gamma^{\mathrm{opt}}=\underset{\gamma}{\operatorname{Argmin}} C(\gamma)
$$


This cost function may be used globally, in a frequency range that includes all tones of interest, or separately in multiple frequency bands associated to different tones. It may also be averaged over several sensors.

\section{Automatic Selection of Tones}

Whether to include or not a tone in the reconstruction formula (8) can be solved by testing the following hypothesis:

$$
H_{0}: c_{k i}=0 \quad \text { for all } \quad i \in\{0, \ldots, I-1\}
$$

for each candidate $k$ in the list. It is proved in the Appendix that the null hypothesis $H_{0}$ is to be rejected if the mean square of the Fourier coefficients is above a given threshold, namely,

$$
\underbrace{\frac{1}{I} \sum_{i=0}^{I-1}\left|c_{k i}\right|^{2}}_{\left\langle\left|c_{k i}\right|^{2}\right\rangle}>\underbrace{\chi_{2 I_{\mathrm{eff}}, 1-p}^{2} \frac{S_{\mathrm{BL}}\left(\alpha_{k} \bar{f}\right) B_{w}}{2 I_{\mathrm{eff}}}}_{t_{k}}
$$

at significance level $p$. In the above equation, $\chi_{2 I_{\mathrm{eff}}, 1-p}$ is the $1-p$ quantile of the chi-square with $2 I_{\text {eff }}$ degrees of freedom, and $S_{\mathrm{BL}}$ $\left(\alpha_{k} \bar{f}\right)$ is the spectral baseline introduced in Sec. III.A and evaluated at frequency $\alpha_{k} \bar{f}$, with $\bar{f}=\sum_{t=0}^{L-1} \dot{\theta}(t) /(2 \pi L)$ the average rotation frequency (this evaluation is easily achieved by interpolation because $S_{\mathrm{BL}}$ is, by construction, a smooth function). $B_{w}=\sum_{t} w^{2}(\gamma t) /$ $\left(\sum_{t} w(\gamma t)\right)^{2}$ is a measure of the spectral bandwidth of kernel $w$ proportional to $\gamma$ (for instance, $B_{w}=1.5 \gamma$ for the Hann window) and

$$
\begin{aligned}
I_{\mathrm{eff}} & =\frac{I}{\rho(0)^{2}+2 \sum_{i=1}^{I-1}\left(1-\frac{i}{I}\right) \rho(i \Delta)^{2}} \leq I \text { with } \\
\rho(\tau) & =\frac{\sum_{t} w(t) w(t-\tau)}{\sum_{t} w^{2}(t)}
\end{aligned}
$$

is the effective number of snapshots, i.e., the number of independent Fourier coefficients in the set $\left\{c_{k i} ; i=2, \ldots, I\right\}$ (see, e.g., [30]).

By using the notations given under the braces in Eq. (19), one has the modified reconstruction formula

$$
\hat{p}(t)=\sum_{k \in \mathcal{A}} \hat{c}_{k}(t) e^{j \alpha_{k} \theta(t)} 1_{\left[\left\langle\left|c_{k}\right|^{2}\right\rangle>t_{k}\right]}
$$

with 1 the indicator function. Consequently, the estimation variance (see Appendix) now becomes

$$
\mathbb{V}\{\hat{p}(t)\}=B_{w} \sum_{k \in \mathcal{A}} S_{\mathrm{BL}}\left(\alpha_{k} \bar{f}\right) 1_{\left[\left\langle\left|c_{k}\right|^{2}\right\rangle>t_{k}\right]} \simeq B_{w} S_{n} N_{p}
$$

where the last approximation holds when the spectral baseline is almost flat, $S_{\mathrm{BL}}(f) \simeq S_{n}\left(S_{n}\right.$ being the noise PSD), and $N_{p}$ stands for the number of significant tones retained in the reconstruction. Clearly, $N_{p}$ has to be as sharp as possible (through setting the value of the quantile $p$ ) in order to control the estimation variance, yet without missing significant harmonics which would then introduce bias errors in the estimate $\hat{p}(t)$.

\section{Simultaneous Tone Extraction from Multiple Measurements}

So far, the proposed extraction method applies on a single signal. If several signals were recorded simultaneously, it would apply on each of them, independently. Such an approach would not take into account that the same tones may be shared by several channels. To fully exploit this property, a specific treatment is required.
The counterpart of model (2) in the multichannel case is

$$
\begin{aligned}
y_{m}(t) & =p_{m}(t)+x_{m}(t), \quad t=0,1,2, \ldots, L-1, \\
m & =1, \ldots, M
\end{aligned}
$$

with $M$ the number of channels, and

$$
p_{m}(t)=\sum_{k \in \mathcal{A}} c_{k}^{m}(t) e^{j \alpha_{k} \theta(t)}
$$

the tonal part of the $m$ th channel, which shares the same orders $\left\{\alpha_{k} ; k \in \mathcal{A}\right\}$ with all other channels. The time-varying Fourier coefficients of the $m$ th channel are then calculated as

$$
c_{k i}^{m}=\frac{1}{\phi_{i}} \sum_{t \in \mathbb{Z}} y_{m}(t) e^{-j \alpha_{k} \theta(t)} w(\gamma(t-i \Delta)) \dot{\theta}(n)
$$

The aim is now to seek spatial filters that, for each order $\alpha_{k}$, transform the Fourier coefficients $c_{k i}^{m}$ into a reduced set of uncorrelated latent variables $z_{k i}^{l}, l=1, \ldots, \tilde{M}<M$, which capture their communalities and, next, to apply the proposed tonal extraction algorithm on each $z_{k i}^{l}$ independently. Let $\boldsymbol{C}_{k}$ denote the $M \times I$ matrix $\left[\boldsymbol{C}_{k}\right]_{m i}=c_{k i}^{m}$ and $\boldsymbol{Z}_{k}$ the $\tilde{M} \times I$ matrix $\left[\boldsymbol{Z}_{k}\right]_{l i}=z_{k i}^{l}$. One seeks the $\tilde{M} \times$ $M$ transformation matrix $\boldsymbol{B}$ such that $\boldsymbol{Z}_{k}=\boldsymbol{B}_{k} \boldsymbol{C}_{k}$ and the $M \times \tilde{M}$ regression matrix $\boldsymbol{A}_{k}$ such that $\boldsymbol{A}_{k} \boldsymbol{Z}_{k}$ is as close as possible to the original $\boldsymbol{C}_{k}$. In the mean-square sense, $\boldsymbol{A}_{k}$ and $\boldsymbol{B}_{k}$ are therefore the minimizers of the Frobenius norm

$$
\left\|\boldsymbol{C}_{k}-\boldsymbol{A}_{k} \boldsymbol{B}_{k} \boldsymbol{C}_{k}\right\|^{2}
$$

where the product $\boldsymbol{A}_{k} \boldsymbol{B}_{k}$ is of reduced-rank $\tilde{M}$. The solution of the above problem is returned by $\boldsymbol{A}_{k}=\boldsymbol{B}_{k}^{H}=\left(\boldsymbol{v}_{k, 1}, \ldots, \boldsymbol{v}_{k, \tilde{M}}\right)$ with $\boldsymbol{v}_{k, l}$ the $l$ th eigenvector of $\boldsymbol{C}_{k} \boldsymbol{C}_{k}^{H} / I$ [31].

The next step is to select the value of $\tilde{M}$ by running the test of significance of Sec. III.C on the eigenvalues $\lambda_{k, l}$ attached to the eigenvectors $\boldsymbol{v}_{k, l}, l=\overline{1, \ldots}, M$. In this case, the spectral baselines to which they have to be compared are returned by

$$
\lambda_{\mathrm{BL}, l}(f)=\mathcal{F}\left\{\boldsymbol{v}_{k, l}^{H}\left(\frac{1}{I^{\prime}} \boldsymbol{Y}(f) \boldsymbol{Y}(f)^{H}\right) \boldsymbol{v}_{k, l}\right\}
$$

evaluated at $f=\alpha_{k} \bar{f}$, where $\mathcal{F}$ stands for the robust smoother introduced in Sec. III.A and $[\boldsymbol{Y}(f)]_{m, i}$ for the STFT coefficient of

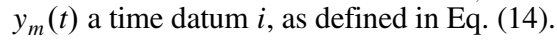

The overall transformation can be resumed as

$$
\tilde{\boldsymbol{C}}_{k}=\underbrace{\left(\sum_{l=1}^{M} 1_{\left[d_{k, l}>t_{k, l}\right]} \boldsymbol{v}_{k, l} \boldsymbol{v}_{k, l}^{H}\right)}_{\mathbb{P}_{k}} \boldsymbol{C}_{k}
$$

with $t_{k, l}=\chi_{2 I_{\text {eff }}, 1-p}^{2} \lambda_{\mathrm{BL}, l}\left(\alpha_{k} \bar{f}\right) B_{w} /\left(2 I_{\text {eff }}\right)$ and $\mathbb{P}_{k}$ a projector in a subspace of dimension $\tilde{M}=\sum_{l=1}^{M} 1_{\left[d_{k, l}>t_{k, l}\right]}$. The remaining index $k$ stresses the fact that the transformation is applied for each order $\alpha_{k}$. The common tonal components are eventually reconstructed in the time domain from the inversion of $\tilde{\boldsymbol{C}}_{k}$ based on Eq. ( $\left.\underline{8}\right)$.

\section{Numerical Experiments}

This section aims at validating the proposed methodology on numerical examples. A synthetic signal is simulated according to the model described by Eqs. (2) and (3). The signal length is $10^{5}$ samples and the sampling frequency is normalized to $1 \mathrm{~Hz}$. The orders are defined as $\alpha_{k}=k, k=1, \ldots, K$, so as to produce $K=|\mathcal{A}|$ harmonic components. The angular function is defined through its time derivative, $\dot{\theta}(t)=2 \pi f_{0}+\varepsilon(t)$, where $f_{0}$ is the central frequency and $\epsilon(t)$ is a first-order autoregressive process, 


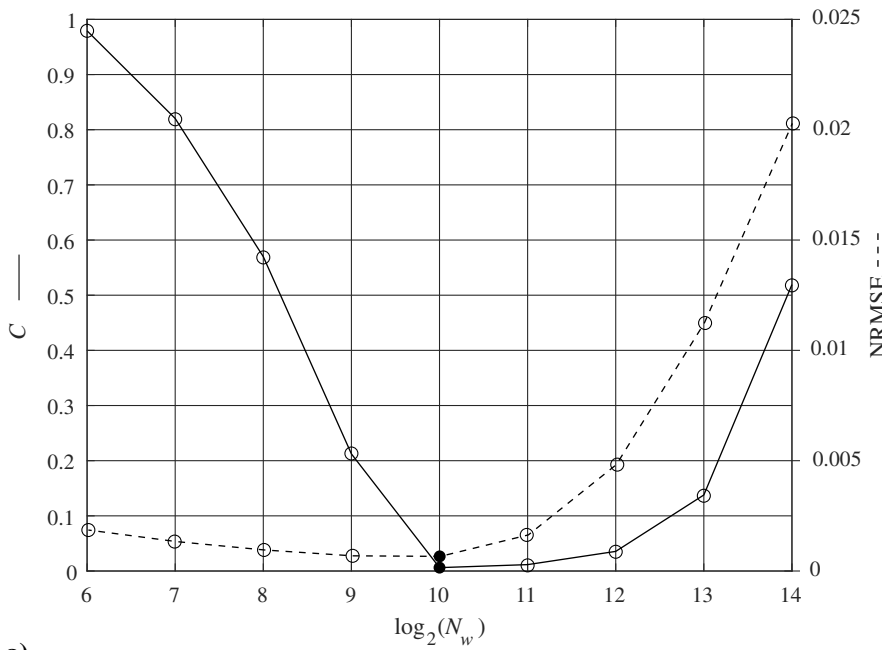

a)

Fig. 1 a) Estimation of the optimal bandwidth $\gamma=1 / N_{w}$ by searching for the minimum of the cost function given by Eq. (16) (continuous line, left scale) and comparison with the normalized root-mean-square error (dotted line, right scale). Positions of the minima are indicated by black bullets. b) Normalized root-mean-square error as a function of the dimension of the projector $\mathbb{P}_{k}$ in Eq. (28) for different subspace dimensions $D=1,2, \ldots, 10$. Positions of the minima of the NRMSE are indicated by black bullets and solutions of the automatic selection by stars.

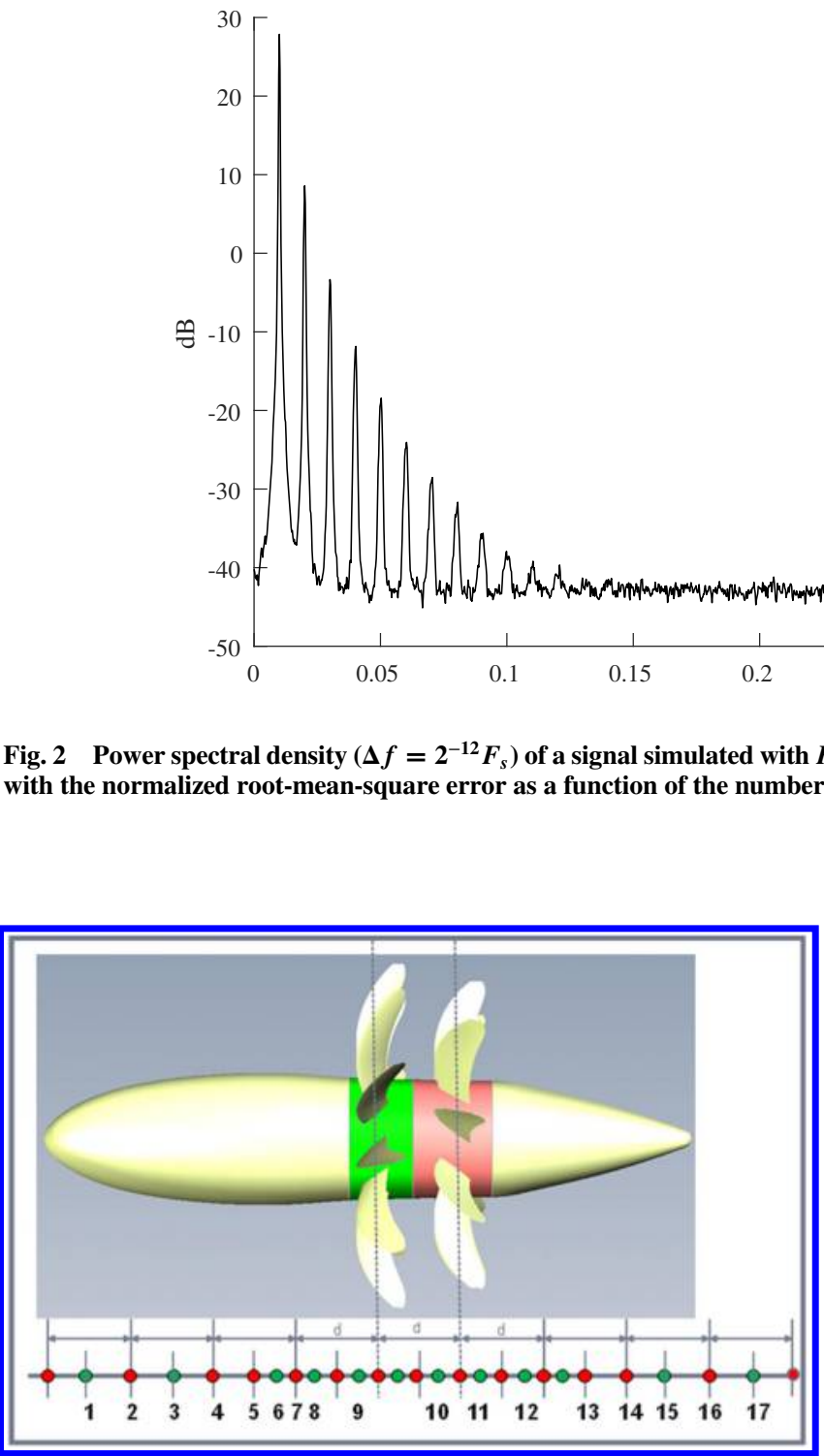

Fig. 3 Schematic of the CROR with the acoustic array. Sensors used in

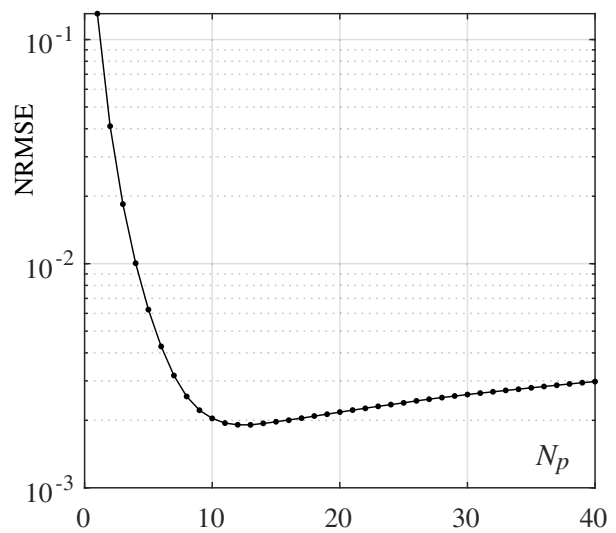

Fig. 2 Power spectral density $\left(\Delta f=2^{-12} F_{s}\right)$ of a signal simulated with $K=40$ harmonics in the presence of background noise (SNR $\left.=40 \mathrm{~dB}\right)$ together with the normalized root-mean-square error as a function of the number $N_{p}$ of extracted tones. the analysis are numbered from 1 to 17. $\epsilon(t)=0.999 \epsilon(t-1)+\nu(t)$, driven by stationary white Gaussian noise $\nu(t)$. The standard deviation of $\dot{\theta}(t)$ is about one per mil (this is enough to invalidate methods that do not explicitly consider angle rather than time as the descriptive variable, even though the method proposed in this paper could handle much larger fluctuations). The time-varying Fourier coefficients of Eq. (3) are synthesized as $c_{k}(t)=c_{0 k}\left(1+\epsilon_{k}(t)\right)$, where $c_{0 k}$ is a deterministic value and $\epsilon_{k}(t)=0.999 \epsilon_{k}(t-1)+\eta_{k}(t)$ with $\eta_{k}(t)$ 's a set of mutually independent stationary white Gaussian noises with complex values [also independent of $\nu(t)]$. The standard deviation of $c_{k}(t)$ is about 0.1 . Finally, the simulated tonal part $p(t)$ is obscured with stationary white Gaussian noise $n(t)$ according to a specified SNR.

The first experiment is to evaluate the capability of the scheme introduced in Sec. III.B to automatically set the bandwidth of the STAFS. The signal is simulated with a single harmonic $[K=1$ in Eq. (3)], with $f_{0}=0.05 F_{s}$ and $c_{0}=1 / 2$. The SNR is $40 \mathrm{~dB}$. The STAFS is used with a Hann window with $1 / 4$ overlap. The spectral baseline (Sec. III.A) is estimated with a frequency resolution of 


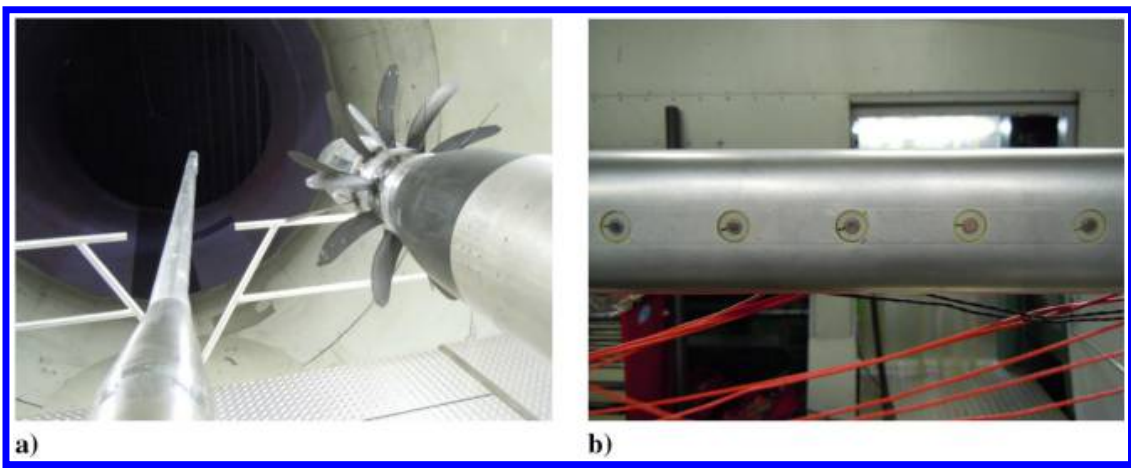

Fig. 4 a) CROR (rearview) with the acoustic array; b) flush-mounted microphones.
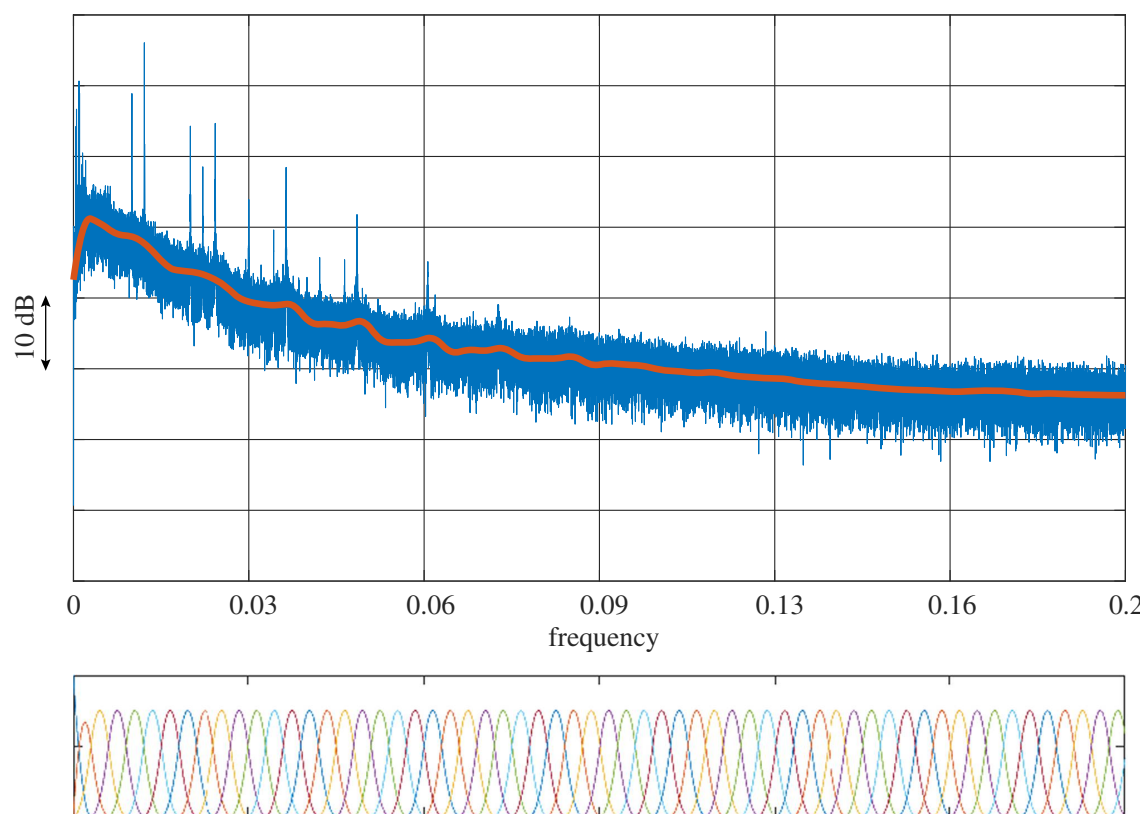

Fig. 5 Illustration of the spectral baseline (arbitrary units) estimated from robust regression of the periodogram (top) on a basis of spline functions (bottom) with bandwidth $\Delta f=5 \times 10^{-3} F_{s}$.

0.015. The cost function (16) is then evaluated for various bandwidths and compared with the normalized root mean square error

$$
\mathrm{NRMSE}=\frac{\|p(t)-\hat{p}(t)\|}{\|p(t)\|}
$$

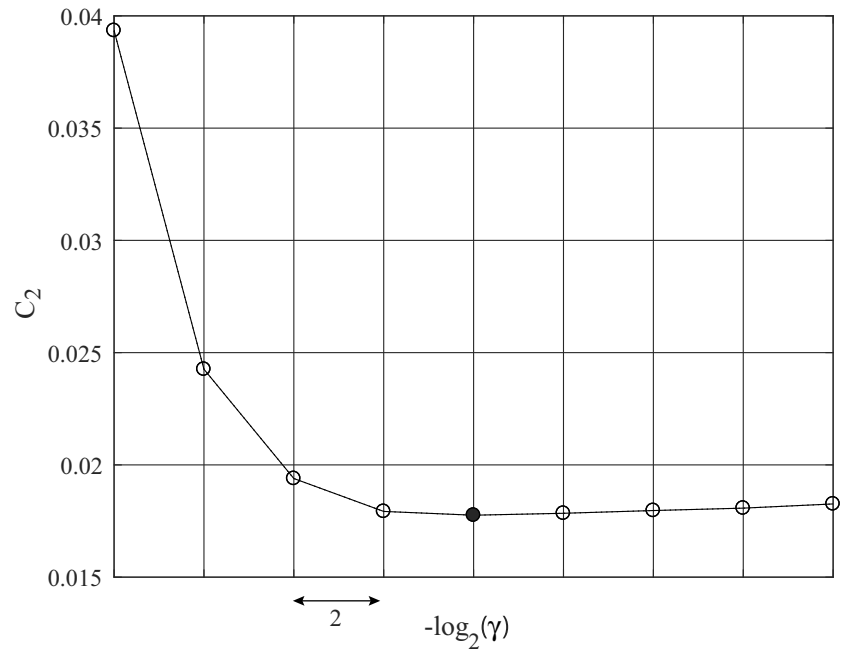

Fig. 6 Estimation of the optimal kernel bandwidth $\gamma$ by searching for the minimum of the cost function $C_{2}(\gamma)$. where $\hat{p}(t)$ stands for the estimated tonal part for a given bandwidth $\gamma$. As seen in Fig. 1a, the cost function $C(\gamma)$ and the NRMSE both indicate the same optimum $\gamma^{\mathrm{opt}}=2^{-10} F_{s}$, which is also close to the bandwidth of the autoregressive process used to simulate the timevarying Fourier coefficient of the signal.

The second experiment is to test the automatic selection of tones of Sec. III.C. The signal is simulated with $f_{0}=0.01 F_{s}$ and $K=40$ harmonics with $c_{0 k}=1 /\left(2 k^{3}\right)$. The SNR is $40 \mathrm{~dB}$, so that the 12 th harmonic is virtually indistinguishable from background noise when a spectral analysis with $\Delta f=2^{-12} F_{s}$ is performed (see Fig. 2). The STAFS setting is as before with the optimal bandwidth just found above. Figure 2 displays the NRMSE as a function of the number $N_{p}$ of tones retained in the extraction. Whereas the minimum NRMSE is for $N_{p}=10$, the automatic selection based on the test given in Eq. (19) returns the value $N_{p}=12$ with significance level 0.99 . Although there is a minor difference, it is observed that the NRMSE is quite flat in the range $11 \leq N_{p} \leq 14$, so that any value in this range will achieve nearly the same minimum.

The last experiment deals with the automatic selection of the dimension of the subspace that is supposed to contain tones simultaneously observed on $M$ sensors. The signal is simulated with $f_{0}=0.01 F_{s}$ and $K=1 . D$ independent realizations of the timevarying Fourier coefficients $c_{0}(t)$ are projected on $M=10$ sensors by multiplication with an $M \times D$ random matrix. This is repeated for $D=1,2, \ldots, 10$. The experimental results indicate that two different behaviors exist depending on whether the SNR is high or low. On the one hand, for high positive SNRs (not shown here), the reduction 
of the NRMSE due to projection in a subspace of dimension lower than $M$ is negligible, provided that it is not less than the actual dimension $D$. In this configuration, it has been observed that the automatic selection scheme of Sec. IV most often selects the correct subspace dimension and, if not, slightly overestimates it. On the other hand, for low SNRs, the reduction of the NRMSE due to projection can be significant. This is illustrated in Fig. $1 \mathrm{~b}$ for an SNR of $0 \mathrm{~dB}$. It is seen that for $D \geq 4$, the minimum of the NRMSE no longer coincides with the actual subspace dimension. This is because in high noise levels, the introduction of a small bias error can result in large reduction of the variance, thus lowering the overall mean square error. There is therefore a benefit of projecting in a subspace whose dimension is smaller than the actual one. As seen in
Fig. 1b, the proposed automatic selection scheme correctly captures this behavior.

\section{Experimental Validation on CROR Data}

The extraction of tones is now illustrated on aeroacoustic signals recorded on a CROR engine $[\underline{32}, 33]$. This CROR data considered particularly difficult for several reasons [34-36]. First, the signals contain a multitude of tonal components over a wide frequency range due to interactions between the two rotors. This results in a long list of incommensurable frequencies. Second, the measurements are polluted by strong background noise due to the turbulent boundary layer. Third, the tones are randomly modulated. As explained in the
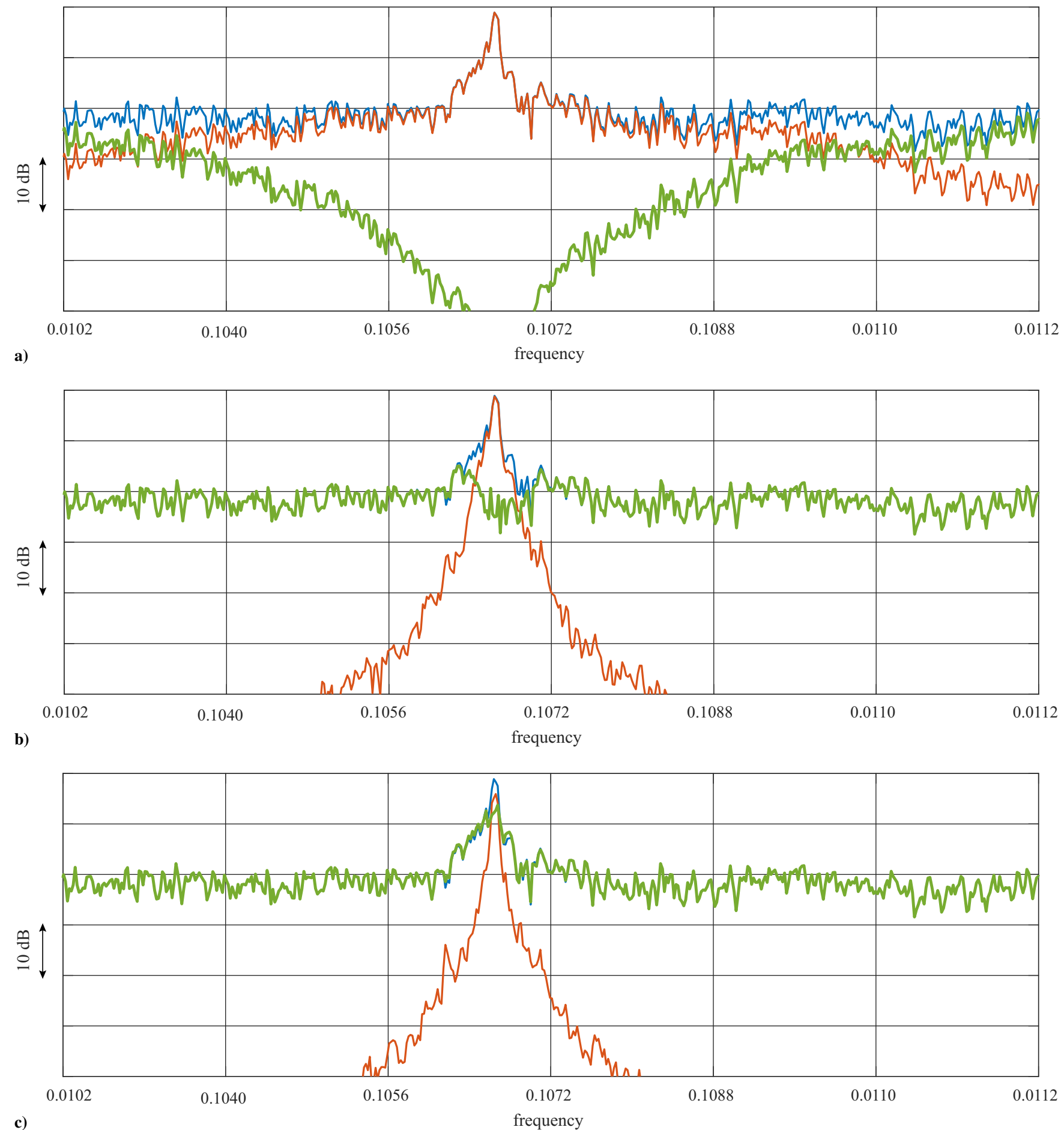

c)

Fig. 7 Power spectral densities (arbitrary units) of the estimated tonal part (red line), the residual part (thick green line), and the total signal (blue line) $\left(\Delta f=4 \times 10^{-4} F_{s}\right)$ with different kernel bandwidths: a) $\gamma=2^{-10}$, b) $\gamma=2^{-14}$, and c) $\gamma=2^{-18}$. 
Introduction, these challenges preclude the use of the synchronous average and are likely to defeat methods based on least squares or model-based tracking filters.

\section{A. Presentation of the Data}

The data were collected during an experimental campaign on a CROR engine placed in a wind tunnel. A linear array was placed $1 \mathrm{~m}$ away from and parallel to the CROR with equispaced flush-mounted microphones. Pressure signals were measured in the near-field of the CROR (see Figs. 3 and 4 ). The instantaneous angles of rotation of the rear and front rotors were measured by two encoders. All results are presented hereafter for Mach speed 0.78 and desynchronized unequal rotor speeds. Frequency values are normalized by the sampling frequency $F_{s}$.

\section{B. Preliminary Analyses}

Spectral analysis reveals that the tonal components pertain to three families (e.g., see blue curve in Fig. 9). The first one comprises the harmonics of the BPF of the rear rotor, denoted by $\left\{k \cdot f_{1}\right.$, $k= \pm 1, \pm 2, \ldots\}$, with $f_{1}$ the (average) rear rotor speed. The second comprises the harmonics of BPF of the front rotor, $\left\{k \cdot f_{2}\right.$, $k= \pm 1, \pm 2, \ldots\}$, with $f_{2}$ the (average) front rotor speed. The third family contains tones produced by the interaction between the two rotors. These are of the form $\left\{m \cdot f_{1}+n \cdot f_{2}, m, n= \pm 1, \pm 2, \ldots\right\}$. Hence, the signal model is in the form of Eq. (4). Specifically, with $\theta_{1}(t)$ and $\theta_{2}(t)$ the angles of rotation of the rear and front rotors,

$$
\begin{aligned}
p(t)= & \underbrace{\sum_{n \in \mathbb{Z}} c_{m 0}(t) e^{j m \theta_{1}(t)}}_{p_{1}(t)}+\underbrace{\sum_{n \in \mathbb{Z}} c_{0 n}(t) e^{j n \theta_{2}(t)}}_{p_{2}(t)} \\
& +\underbrace{\sum_{\substack{m, n) \in \mathbb{Z}^{2} \\
n \neq 0, m \neq 0}} c_{m n}(t) e^{j\left(m \theta_{1}(t)+n \theta_{2}(t)\right)}}_{p_{12}(t)}
\end{aligned}
$$

where $p_{1}(t), p_{2}(t)$, and $p_{12}(t)$ embody the tonal components of the rear and front rotors and their interactions, respectively. Because the two rotors are not cinematically locked, the ratio between the two frequencies $f_{1}$ and $f_{2}$ is not constant. Spectral analysis also reveals that tones do not show off as perfect peaks but are slightly distributed (e.g., see blue curve in Fig. 7). This is partly because the rotor speeds are not constant, yet the shape and width of the peak distribution indicate that other reasons exist. It is believed that periodic acoustical waves experience random modulation when propagating in the presence of turbulence, thus becoming narrowband random. The chopping of the waves by the rotation of the blades typically broadens tones more and more with frequency. These effects also jeopardize the use of the classical synchronous average, at least if used in its original version. The STAFS proposed in this paper allows the unwrapping of these random modulations to some extent and therefore reassigns the corresponding power to the tonal components rather than to the broadband components. This is deemed meaningful as this power originally pertained to the tones before it got distributed in frequency.

As explained in Sec. III, the first step in the automatic extraction of tones is to estimate the baselines of the spectra. These will be used to automatically set up the kernel bandwidth of the STAFS and to detect significant tones. Figure 5 gives one example of a spectral baseline obtained from the proposed robust regression on a basis of spline functions with spectral bandwidth of $\Delta f=5 \times 10^{-3} F_{s}$. It is noteworthy that the spectral baseline estimated in this way is virtually insensitive to the presence of tones.

The next step is to automatically set up the kernel bandwidth $\gamma$ of the short-time windows in the STFT transform. As explained in Sec. III.B, this requires a balance between a value short enough to properly capture the aforementioned signal modulations and long enough to improve the extraction capability (the dynamic range of the extracted tones is proportional to $1 / \gamma$ ). The cost function (16) shows an optimal value around $\gamma=2^{-14} F_{s}$ (see Fig. 6). The rational beyond this criterion is illustrated by Fig. 7. A too short window introduces excessive removal of the spectral components in the vicinity of the peaks, thus producing artificial gaps in the spectrum of the residual signal after extraction of estimated tones. On the opposite, a too long window is not able to capture the spectral spread of the peaks and part of the tones is still present in the residual signal.

Another question is whether the tones should be extracted independently on each sensor or simultaneously on all sensors. Because the tonal components correspond to acoustical waves with a high correlation length, a coherence is expected between the microphones of the array. Therefore, the multivariate approach is deemed more relevant. This fact is experimentally confirmed by comparing the extraction results obtained from the two strategies in Figs. 8 and 9 . It is seen that the multivariate approach is able to extract tones of small magnitude that are not detected as significant with the univariate approach. The multivariate approach detects them because
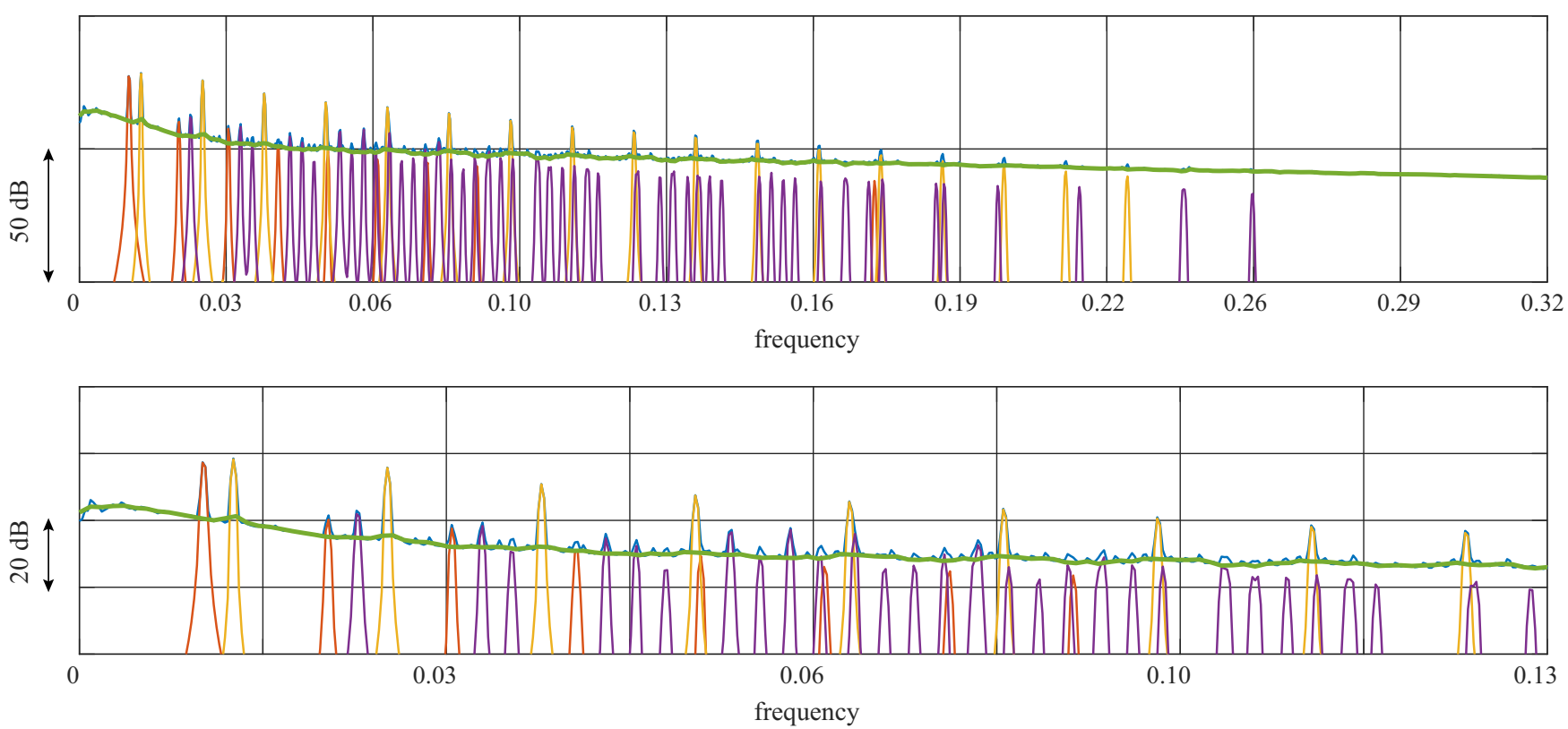

Fig. 8 Separation from the univariate strategy on sensor \#11. Power spectral densities (arbitrary units) of the estimated tonal part attached to the rear rotor (red line), the front rotor (orange line), the interactions (violet line), and of the residual (thick green line) and total (blue line) signals $\left(\Delta f=4 \times 10^{-4} F_{s}\right)$. The bottom view is an excerpt of the top view. 

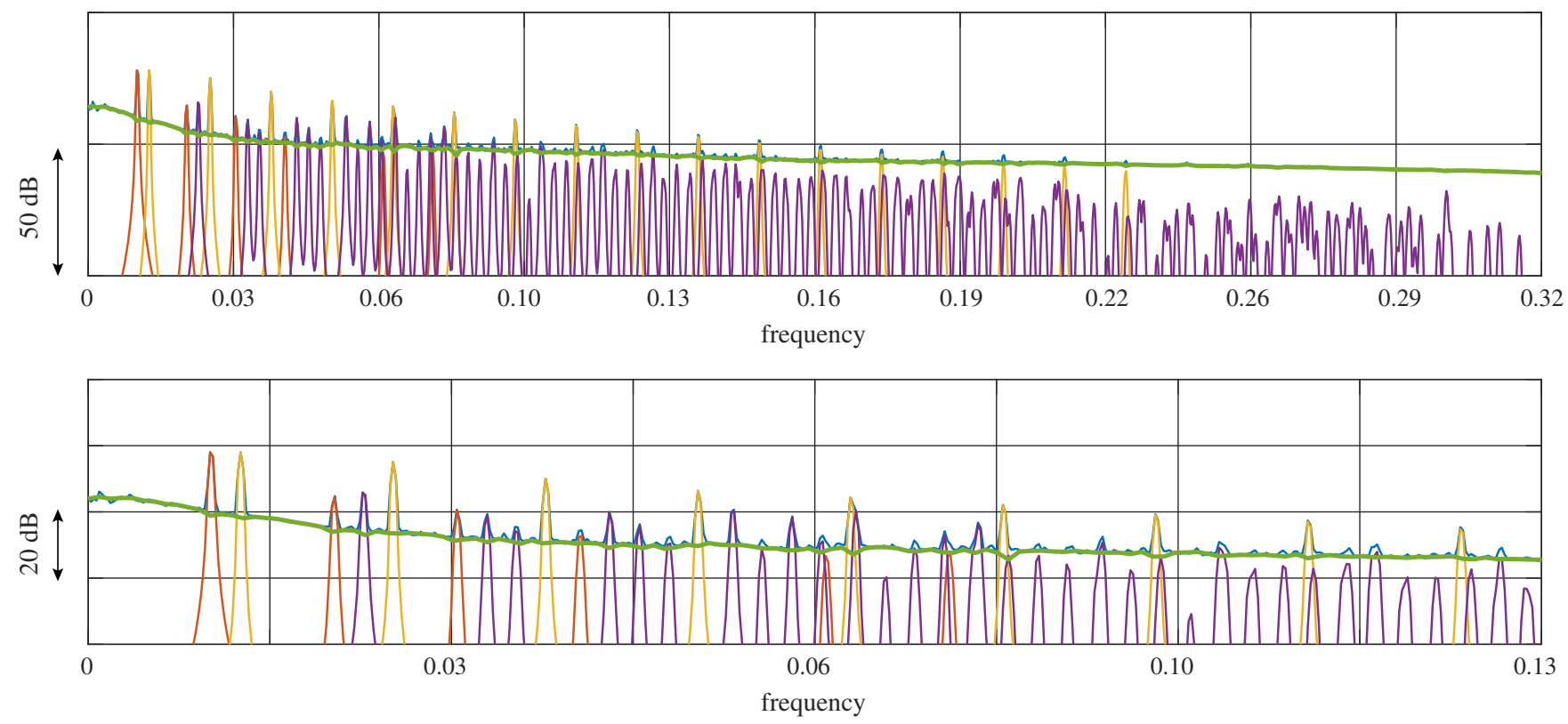

Fig. 9 Separation from the multivariate strategy on sensor \#11. Power spectral densities (arbitrary units) of the estimated tonal part attached to the rear rotor (red line), the front rotor (orange line), the interactions (violet line), and of the residual (thick green line) and total (blue line) signals $\left(\Delta f=4 \times 10^{-4} F_{s}\right)$. The bottom view is an excerpt of the top view.

they actually exist on all sensors, possibly with high magnitude on some of them.

For the sake of the comparison, Fig. 10 displays the results of synchronous averaging the two shafts separately. The signal was first resampled in the angular domain of the shaft of interest and, after being synchronously averaged, resampled back to the time domain. It is noteworthy that the extraction of the interaction tones was not possible as these are not synchronous to either shaft. It is seen that the power of the tones is largely underestimated and, consequently, the complementary residual part still contains a large portion of the peaks. This is because simple synchronous averaging is not able to track the random modulations of the tones (a refined version could be used as in Ref. [9], yet it would not be able to extract the interaction tones anyway). The extraction of a tone by synchronous averaging is actually very similar to the use of the proposed method with a too small bandwidth, as illustrated in Fig. 7c. For another point, synchronous averaging is unable to make the difference between insignificant tones and background noise. This is why all harmonics appear in Fig. 10, even those that have been classified as indistinguishable from background noise by the proposed method (e.g., harmonic orders of the BPF1 higher than 18th).

\section{Analysis of Separation Results}

Based on the above observations, it was decided to use the multivariate strategy with $\gamma=2^{-14}$. With this setting, the extraction of a tone in a signal consisting of 1.9 samples took a fraction of a second with an Intel Core i7-4810MQ processor. The statistical threshold used to detect significant tones is set to a risk of 5\%. The maximum number of eigenvalues of the cross-spectral matrix is equal to the number of sensors $(M=17)$. Figure 11 displays the results of the automatic selection of tones in the three families. The ordinates
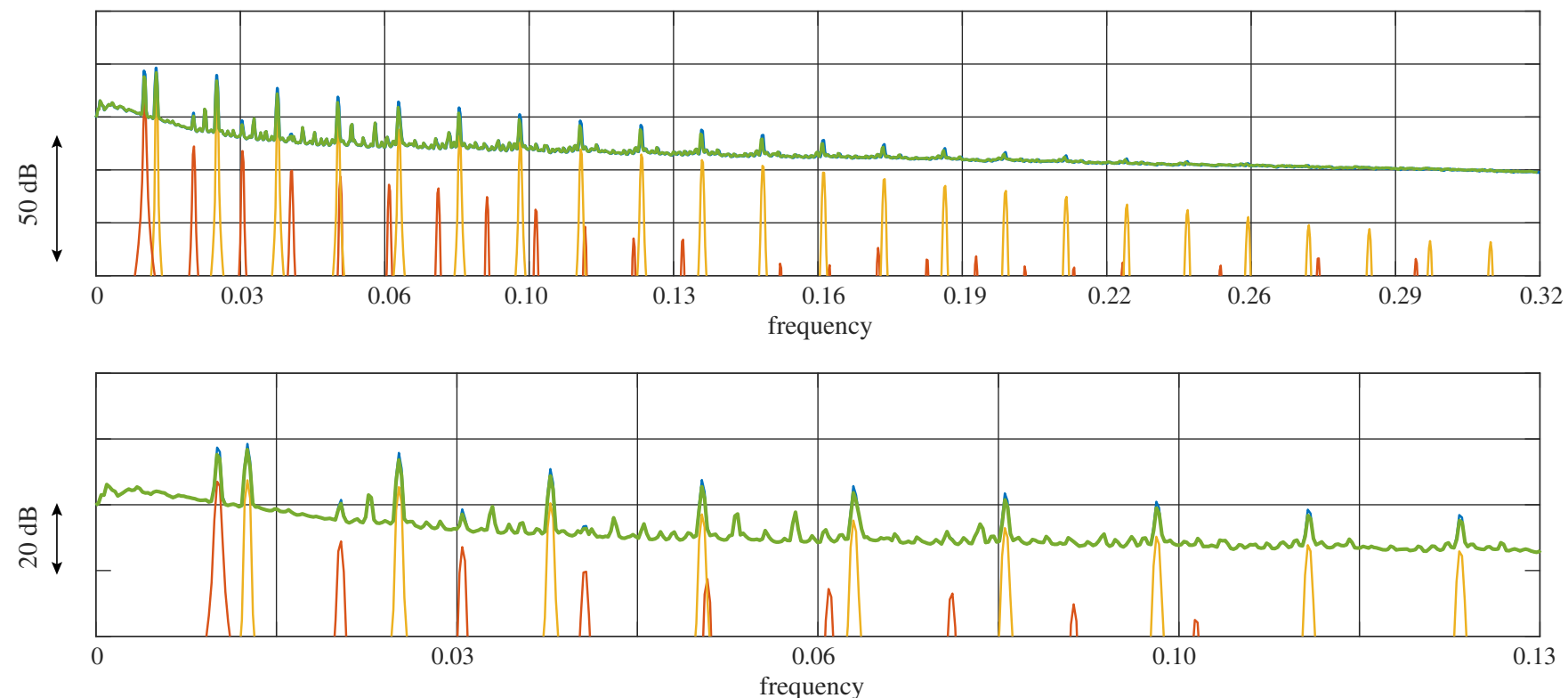

Fig. 10 Separation from simple synchronous averaging on sensor \#11. Power spectral densities (arbitrary units) of the estimated tonal part attached to the rear rotor (red line), the front rotor (orange line), and of the residual (thick green line) and total (blue line) signals $\left(\Delta f=4 \times 10^{-4} F_{s}\right)$. The bottom view is an excerpt of the top view. 

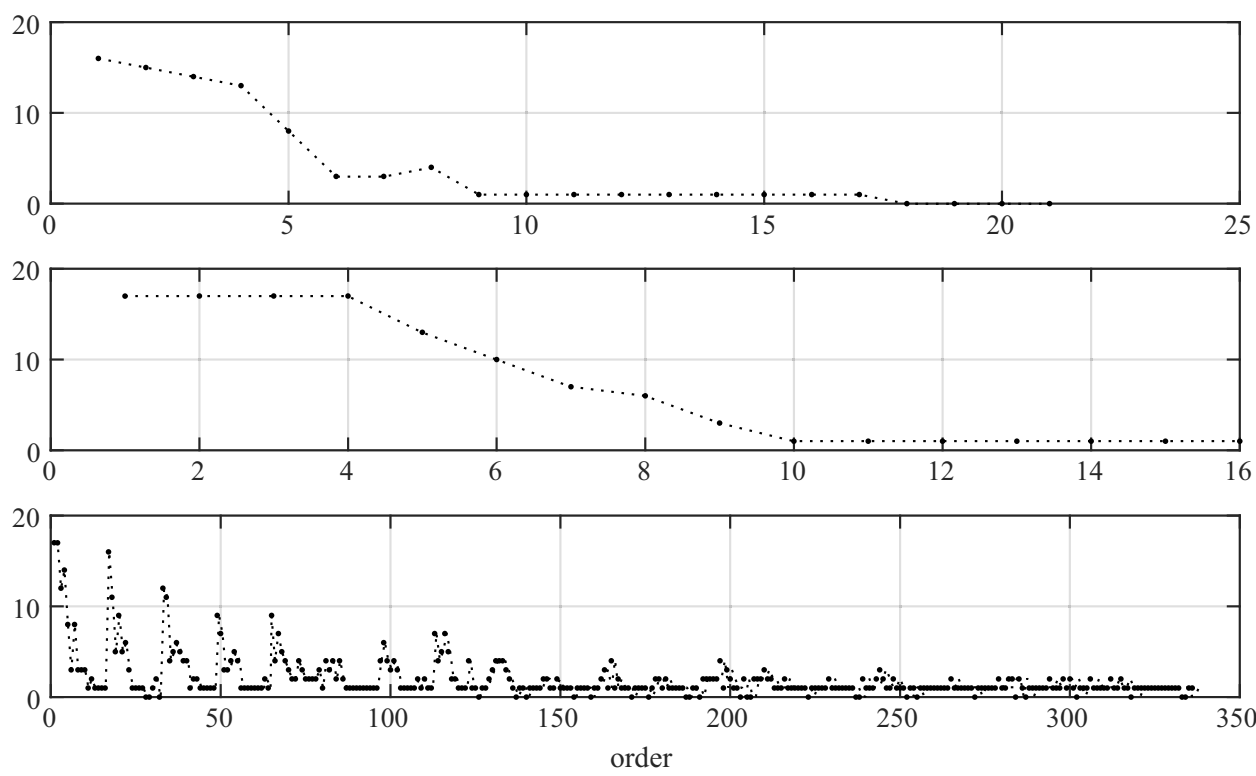

Fig. 11 Automatic selection of the significant tones in the families related to the rear rotor (top), the front rotor (middle), and the interactions (bottom). The ordinates correspond to the number of degrees of freedom required to model each tone. A value of zero means that the tone is not selected.

correspond to the number of degrees of freedom required to model each tone, as returned by the number of significant eigenvalues at the corresponding frequency in the spectral matrix (see Fig. 12). The high number of degrees of freedom indicates the presence of random modulation (in theory, a pure tone would require only one degree of freedom). Its decrease as a function of frequency follows the evolution of the SNR. It is noteworthy that most of the tones in the third family are significant, even though their magnitude is actually very small (as seen in Fig. 13, they rapidly move below the noise level).

Visual inspection of the PSDs of all sensors confirms an excellent separation of the tonal and broadband components. The PSDs of the residual signals, after removal of tones, are continuous densities. Careful inspection reveals that some small spectral ripples remain around the extracted tones. As explained above, these are due to the presence of random modulation of the tones (they are particularly noticeable in the first eigenspectra in Fig. 12). Whether they should be assigned to the tonal part or the AT-CS2 part is not clear, because they truly result from interaction between the two parts. In any case, if not taken into account in the first part, they will be in the second one.

The intensity of the tones strongly depends on the spatial location of the sensor. They are clearly stronger on sensors facing the rotor than on sensors located farther upstream. The spatial distribution of the tonal energy is displayed in Fig. 13. As expected, the energy distribution assigned to the rear rotor is slightly shifted behind the energy distribution assigned to the front rotor. The energy distribution of the interactions is distinctly localized behind the front rotor, which is fully consistent with their physical origins.

Finally, Fig. 14 shows the tonal parts reconstructed in the time domain for sensor \#10. It is noteworthy that the tonal parts of the rear and front rotors are periodic, whereas that of the interactions is not because it is composed of incommensurate frequencies. The signal is actually pseudoperiodic, yet because of its rich spectral content it
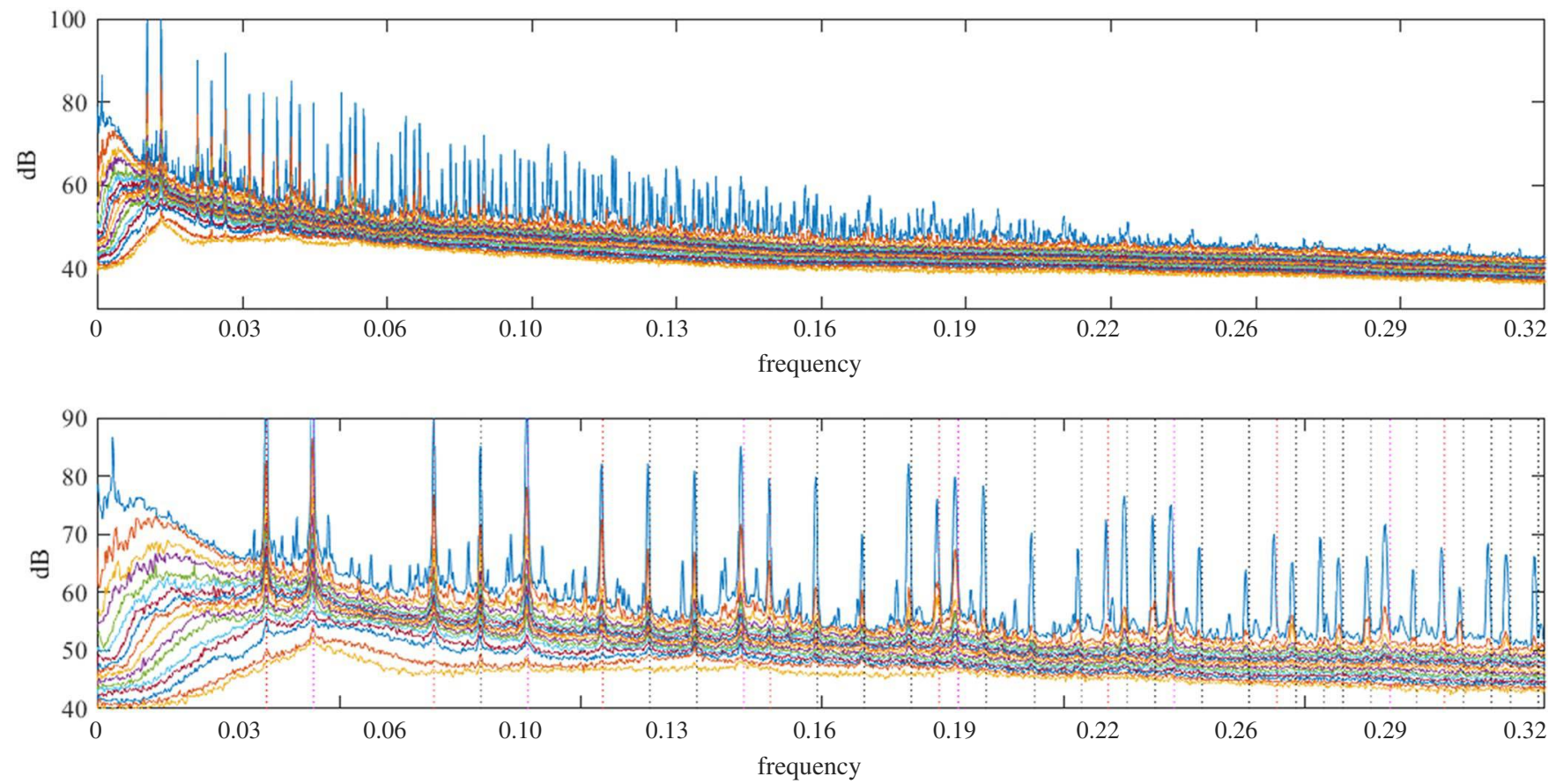

Fig. 12 Eigenspectra of the cross-spectral matrix (arbitrary units). The bottom view is an excerpt of the top view up to $0.1 F_{s}$. Vertical lines indicate theoretical positions of the tones $\left(\Delta f=10^{-5} F_{s}\right)$. 

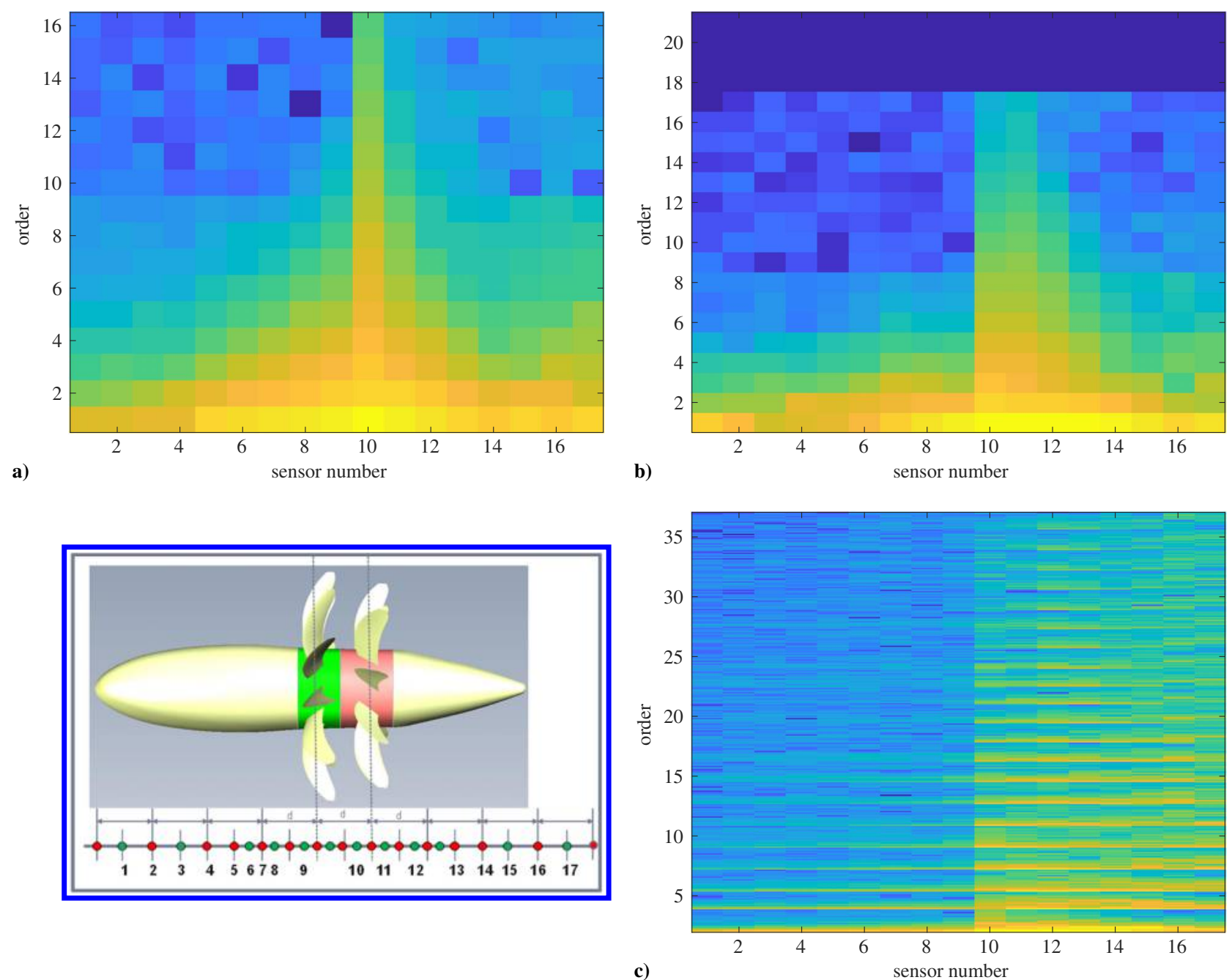

Fig. 13 Power of the extracted tonal components (arbitrary units): a) front rotor, b) rear rotor, and c) their interactions as functions of frequency and space $\left(\Delta f=4 \times 10^{-3} F_{s}\right)$.
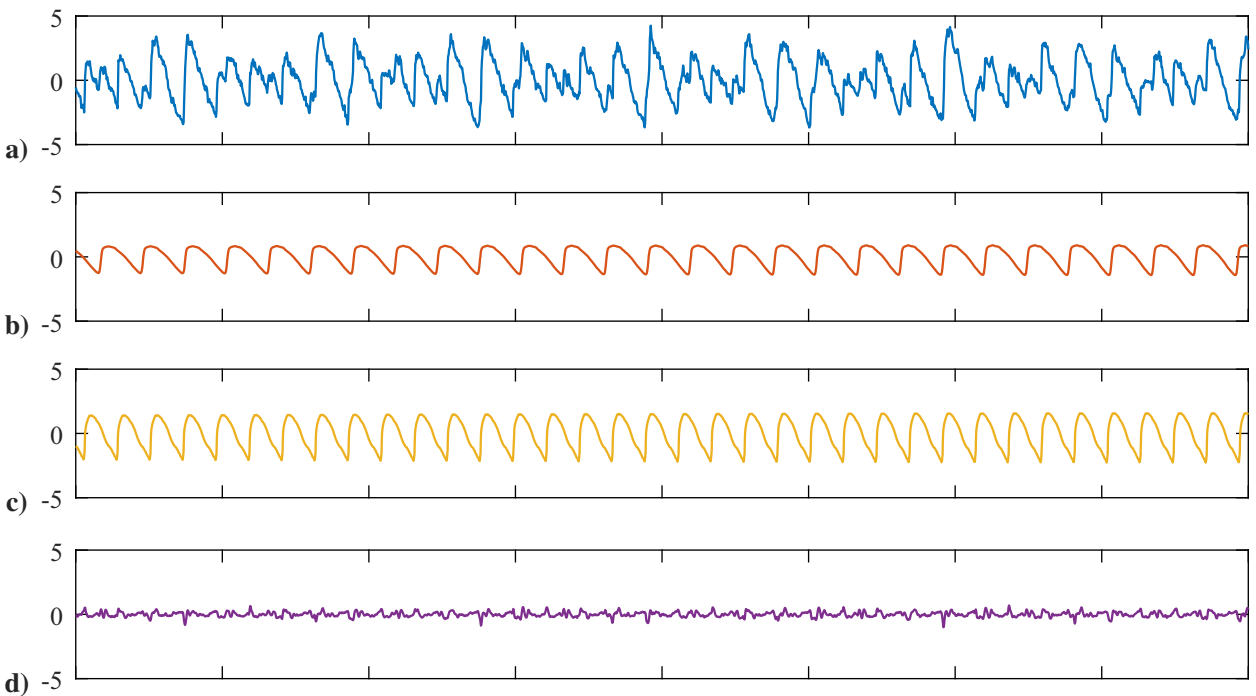

d)

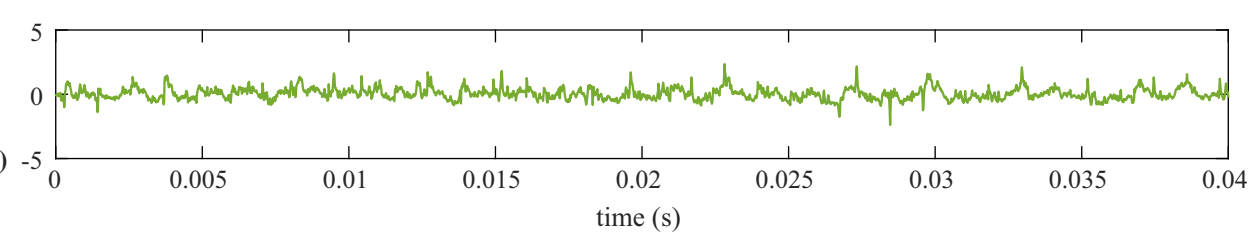

Fig. 14 Extracted time signals (arbitrary units) for sensor \#10 with the same code of colors in Figs. 8 and 9: a) total signal $y(t)$; b) tonal part due to rear rotor $\left.\hat{p}_{1}(t) ; c\right)$ tonal part due to front rotor $\left.\hat{p}_{2}(t) ; \mathrm{d}\right)$ tonal part due to interactions $\left.\hat{p}_{12}(t) ; \mathrm{e}\right)$ residual signal $\hat{x}(t)$. 
looks almost random. It also shows a lower magnitude than the individual contributions of the rear and front rotors. The residual signal obtained after removal of tonal components eventually ends up broadband and purely random.

\section{Conclusions}

A method has been proposed to automatically extract tones from aeroacoustic signals. Compared with the state-of-the-art, the proposed solution is flexible enough to extract in reasonable time very large numbers of tones, even when distributed at incommensurate frequencies. When concerned with the sequential extraction of tones in a single signal, it offers a valuable alternative to the Vold Kalman filter, with similar complexity and performance. Because it is rooted in the theory of angle-time cyclostationary processes, which is well suited to modeling aeroacoustic signals, it is able to compensate to some extent for the presence of moderate random modulations. A special effort has been made to relieve as much as possible of the user expertise by introducing data-driven strategies for setting up parameters. The proposed methodology has proved very efficient when processing several datasets, including the CROR data that are known for being challenging. As a byproduct, the extraction of tones also returns the broadband contribution of the signals. The latter is just as likely to contain relevant information. For instance, it typically embodies turbulent pressure fluctuations modulated by the rotor speed or the blade-passing frequency. The extraction of these components from the remaining background noise is also possible from the theory of angle-time cyclostationary processes. It will be the topic of a sequel in a companion paper.

\section{Appendices}

\section{A.1. Near-Perfect Reconstruction of the STAFS}

Let us consider the monochromatic case $y(t)=c(t) e^{j \alpha \theta(t)}$. On the one hand, the Fourier coefficient returned by formula ( $\underline{5}$ ) reads

$$
c_{i}=\frac{\sum_{t \in \mathbb{Z}} c(t) w(\gamma(t-i \Delta)) \dot{\theta}(t)}{\sum_{t \in \mathbb{Z}} w(\gamma(t-i \Delta)) \dot{\theta}(t)}
$$

Assuming that the Fourier coefficient $c(t)$ has variations slow enough so that it can be considered nearly constant in the time interval covered by $w(\gamma(t-i \Delta))$ (assumption $\mathrm{H} 3)$, one has $c_{i} \simeq c(i \Delta)$ with exact equality when $c(t)$ is a constant. On the other hand, the reconstruction formula (8) requires that $\hat{p}(t)=p(t)$, which is fulfilled provided that

$$
c(t)=\kappa \sum_{i=0}^{I-1} c_{i} w(\gamma(t-i \Delta))
$$

The meaning of the above equation is that $c(t)$ can be interpolated with kernel $w(t)$. This is again compliant with assumption $\mathrm{H} 3$. The special case where $c(t) \equiv c$ is a constant returns $c_{i} \simeq c$ and the condition

$$
1=\kappa \sum_{i=0}^{I-1} w(\gamma(t-i \Delta))
$$

This requires $\{w(\gamma(t-i \Delta)) ; i=0, \ldots, I-1\}$ to partition the unity (which can be achieved at least for $N_{w} / 2 \leq t<L-N_{w} / 2$ ) and the constant $\kappa$ to be defined as in Eq. (8). It places a constraint on the value of the stride $\Delta$, which must be small enough. For instance, with a Hann window of length $N_{w}, \Delta$ may be equal to $N_{w} / 4$. Note that, given an arbitrary positive window, say, $v(t)$, condition (A3) can always be forced by setting

$$
w(t)=\frac{v(t)}{\sum_{i=0}^{I-1} v(\gamma(t-i \Delta))}
$$

provided that $\Delta$ is small enough so that $\sum_{i=0}^{I-1} v(\gamma(t-i \Delta)) \neq 0$ everywhere.

It is noteworthy in passing that, under these conditions, the shorttime Fourier coefficients (5) lend themselves to the recovery of a global-i.e., constant-Fourier coefficient through the simple time average

$$
c=\frac{1}{I} \sum_{i=0}^{I-1} \hat{c}_{i}
$$

For the sake of comparison with another technique, Fig. A1 displays the frequency gains of the STAFS with a Hann window and of the Vold-Kalman filter. It is seen that the gains are very similar. They

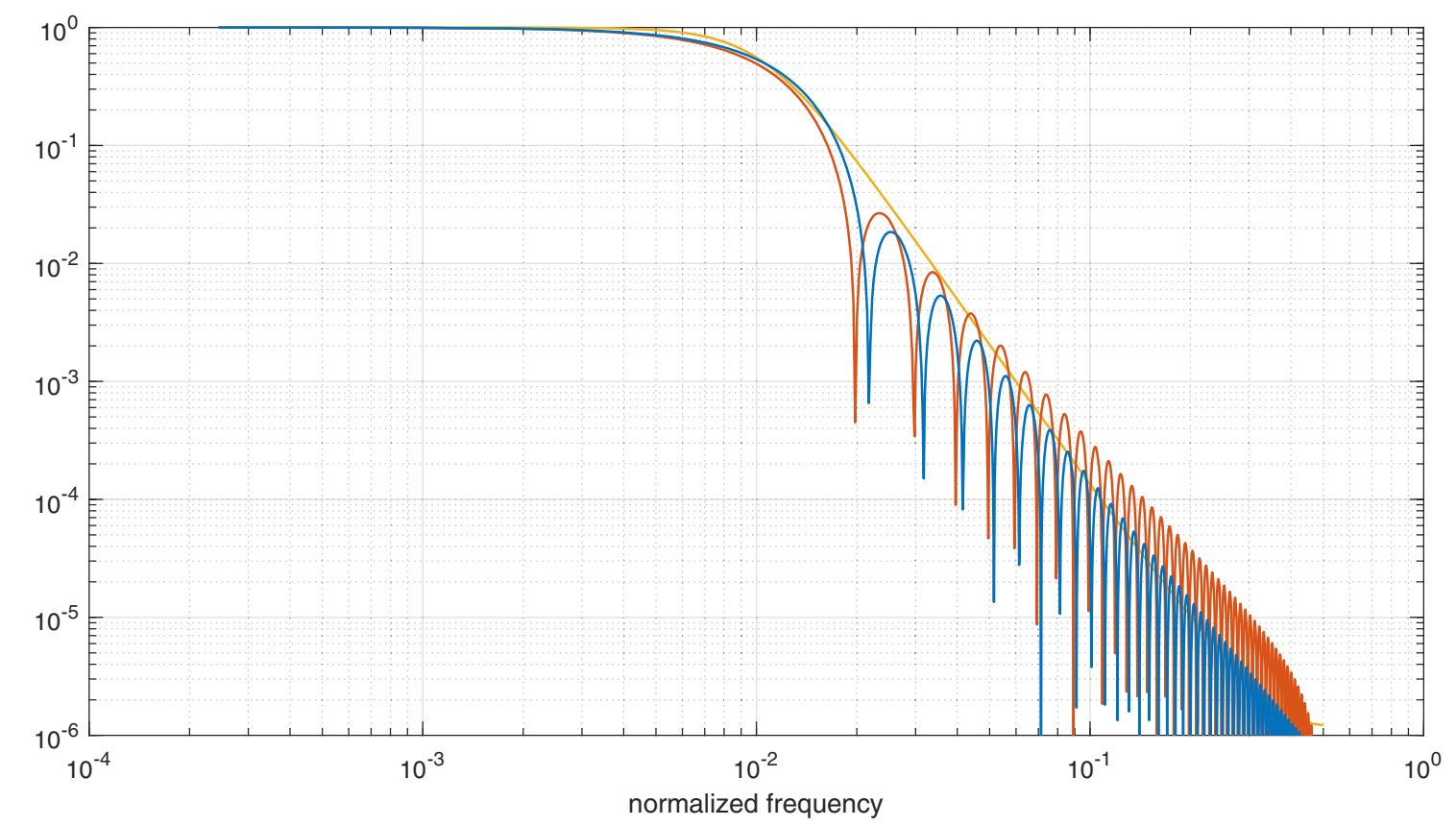

Fig. A1 Comparison of the frequency gains of the STAFS with a Hann window (red) and a Hann window raised to the power 1.2 (blue) with the two-pole Vold-Kalman filter (orange). 
can actually be made arbitrarily close by changing the design of the window $w(t)$, for instance, by raising the Hann window to some power; here a Hann window to the power 1.2 produces nearly the same gain as the two-pole Vold-Kalman filter.

\section{A.2. Bandwidth Selection}

Under mild conditions, it can be reasonably assumed that the $j$ th snapshot $E_{i}\left(f_{k}\right)$ at frequency $f_{k}$ follows a zero-mean complex Gaussian with variance $\sigma^{2}\left(f_{k}\right)$ [37]. Provided that the set of snapshots $i=0, \ldots, I^{\prime}-1$ are independent with respect to both the time and frequency indices $j$ and $k$, the quantities $\left\{\sum_{i=0}^{I^{\prime}-1}\left|E_{i}\left(f_{k}\right)\right|^{2}\right.$; $k \in \mathcal{K}\}$ jointly follows a complex Wishart with an $F \times F$ covariance matrix (with $F=|\mathcal{K}|$ ) composed of the diagonal elements $\sigma^{2}\left(f_{k}\right)$. Now let us make $E_{i}\left(f_{k} ; \gamma\right)$ depend explicitly on a parameter $\gamma$ and search for the value $\gamma^{\text {opt }}$ that makes the observations $\left\{\sum_{i=0}^{I^{\prime}-1} \mid\right.$ $\left.\left.E_{i}\left(f_{k} ; \gamma\right)\right|^{2} ; k \in \mathcal{K}\right\}$ as close as possible to the realizations of the stochastic process defined by the complex Wishart with covariance matrix composed of $S_{\mathrm{BL}}\left(f_{k}\right)$. This amounts to maximizing the likelihood function of the complex Wishart, i.e.,

$$
\begin{aligned}
& \gamma^{\mathrm{opt}}=\operatorname{Argmax}_{\gamma} \Pi_{k \in \mathcal{K}}\left(\exp \left(-\frac{\sum_{i=0}^{I^{\prime}-1}\left|E_{i}\left(f_{k} ; \gamma\right)\right|^{2}}{S_{\mathrm{BL}}\left(f_{k}\right)}\right) \frac{\left(\sum_{i=0}^{I^{\prime}-1}\left|E_{i}\left(f_{k} ; \gamma\right)\right|^{2}\right)^{I^{\prime}-1}}{S_{\mathrm{BL}}\left(f_{k}\right)^{I^{\prime}}}\right) \\
& =\operatorname{Argmin} \underbrace{}_{\gamma} \underbrace{\sum_{k \in \mathcal{K}}\left(\frac{\sum_{i=0}^{I^{\prime}-1}\left|E_{i}\left(f_{k} ; \gamma\right)\right|^{2}}{S_{\mathrm{BL}}\left(f_{k}\right)}-\left(I^{\prime}-1\right) \ln \left(\frac{\sum_{i=0}^{I^{\prime}-1}\left|E_{i}\left(f_{k} ; \gamma\right)\right|^{2}}{S_{\mathrm{BL}}\left(f_{k}\right)}\right)\right)}_{C_{1}(\gamma)}
\end{aligned}
$$

where all constant terms have been removed. Because the so-defined cost function $C_{1}(\gamma)$ is growing with $I^{\prime}$, it is sensible to normalize it. At the same time, care should be taken to make it unbiased. Hence, the aim is to find a modified cost function $C_{2}(\gamma)=C_{1}(\gamma) / I^{\prime}+c$ such that, by convention, $\mathbb{E}\left\{C_{2}(\gamma)\right\}=1$ when $\sum_{i=0}^{I^{\prime}-1}\left|E_{i}\left(f_{k}, \gamma\right)\right|^{2}$ is actually distributed like the assumed complex Wishart. Let define $S_{k}=\sum_{i=0}^{I^{\prime}-1}\left|E_{i}\left(f_{k} ; \gamma\right)\right|^{2} / S_{\mathrm{BL}}\left(f_{k}\right) ; 2 S_{k}$ is seen to follow a chi-squared with $2 I^{\prime}$ degrees of freedom. Therefore, $\mathbb{E}\left\{S_{k}\right\}=I^{\prime}$ and $\mathbb{E}$ $\left\{\ln S_{k}\right\}=\psi\left(I^{\prime}\right)$ with $\psi$ the digamma function and $c=\left(1-1 / I^{\prime}\right)$ $\psi\left(I^{\prime}\right)$. Now, for $I^{\prime} \gg 1$ (as requested by spectral analysis), $\psi\left(I^{\prime}\right) \simeq \ln I^{\prime}$, from which the cost function (16) immediately follows.

\section{A.3. Test of Significance}

Under the null hypothesis (18), the Fourier coefficient $c_{k i}$ is assumed to follow a zero-mean complex Gaussian distribution (the Gaussian distribution is sensible, because it is asymptotically true under mild conditions due to the Central Limit Theorem [37]). Assuming first that the rotation speed is constant, i.e., $\dot{\theta}(t)=\overline{2} \pi \bar{f}$, the variance of $c_{k i}$ is

$$
\mathbb{V}\left\{c_{k i}\right\}=S_{n}\left(\alpha_{k} \bar{f}\right) B_{w}
$$

where $S_{n}\left(\alpha_{k} \bar{f}\right)$ is the theoretical PSD of background noise and $B_{w}=\sum_{t} w(\gamma t)^{2} /\left(\sum_{t} w(\gamma t)\right)^{2}$ is proportional to the spectral bandwidth $\gamma$. Equation (A7) remains a very good approximation when the rotation speed fluctuates slowly enough so that $S_{n}(f) \simeq S_{n}\left(\alpha_{k} \bar{f}\right)$ for all values of $f$ in the span of $\dot{\theta}(t) /(2 \pi)$. With these results, the mean square quantity $2 \sum_{i=0}^{I-1}\left|c_{k i}\right|^{2} /\left(S_{n}\left(\alpha_{k} \bar{f}\right) B_{w}\right)$ follows a chi-squared distribution with $2 I_{\text {eff }}$ degrees of freedom, where $I_{\text {eff }}$ is the equivalent number of independent terms in the set of Fourier coefficients, as given by Eq. $\left\{c_{k i} ; i=0, \ldots, I-1\right\}\left(I_{\mathrm{eff}} \leq I\right.$ due to overlapping of snapshots [30]). Therefore, under the null hypothesis,

$$
\frac{1}{I} \sum_{i=0}^{I-1}\left|c_{k i}\right|^{2} \sim \frac{S_{n}\left(\alpha_{k} \bar{f}\right) B_{w}}{2 I_{\text {eff }}} \chi_{2 I_{\text {eff }}}^{2}
$$

where the $\sim$ sign means "distributed like."

\section{Acknowledgments}

This work was funded by Clean Sky 2 Joint Undertaking, European Union (EU), Horizon 2020, CS2-RIA, and ADAPT project (Grant agreement no. 754881). This work was partly performed within the Labex CeLyA of the Universite de Lyon, in the program "Investissements d'Avenir" (ANR-10-LABX-0060/ANR-16-IDEX0005) operated by the French National Research Agency.

\section{References}

[1] Glegg, S., and Devenport, W., "Chapter 16-Open Rotor Noise," Aeroacoustics of Low Mach Number Flows, edited by S. Glegg, and W. Devenport, Academic Press, New York, 2017, pp. 399-436. https://doi.org/10.1016/B978-0-12-809651-2.00016-3

[2] Magliozzi, B., Hanson, D. B., and Amiet, R. K., "Aeroacoustics of Flight Vehicles: Theory and Practice. Volume 1: Noise Sources," NASA TR 90-3052, 1991.

[3] Hurd, H. L., "Nonparametric Time Series Analysis for Periodically Correlated Processes," IEEE Transactions on Information Theory, Vol. 35, No. 2, 1989, pp. 350-359.

[4] Antoni, J., "Cyclostationarity by Examples," Mechanical Systems and Signal Processing, Vol. 23, No. 4, 2009, pp. 987-1036. https://doi.org/10.1016/j.ymssp.2008.10.010

[5] Jurdic, V., Joseph, P., and Antoni, J., "Investigation of Rotor Wake Turbulence Through Cyclostationary Spectral Analysis," AIAA Journal, Vol. 47, No. 9, 2009, pp. 2022-2030. https://doi.org/10.2514/1.36728

[6] Rémond, D., Antoni, J., and Randall, R., "Instantaneous Angular Speed (IAS) Processing and Related Angular Applications," Mechanical Systems and Signal Processing, Vol. 45, No. 1, 2014, pp. 24-27. https://doi.org/10.1016/j.ymssp.2013.10.015

[7] Braun, S., and Seth, B., "Analysis of Repetitive Mechanism Signatures," Journal of Sound and Vibration, Vol. 70, No. 4, 1980, pp. 513-526. https://doi.org/10.1016/0022-460X(80)90321-1

[8] Behn, M., Pardowitz, B., and Tapken, U., "Separation of Tonal and Broadband Noise Components by Cyclostationary Analysis of the Modal Sound Field in a Low-Speed Fan Test Rig," International Conference of Fan Noise, Aerodynamics, Applications and Systems, 2018.

[9] Klein, R., "Comparison of Methods for Separating Vibration Sources in Rotating Machinery," Mechanical Systems and Signal Processing, Vol. 97, Dec. 2017, pp. 20-32. https://doi.org/10.1016/j.ymssp.2017.03.040

[10] Antoni, J., Ducleaux, N., Nghiem, G., and Wang, S., "Separation of Combustion Noise in IC Engines Under Cyclo-Non-Stationary Regime," Mechanical Systems and Signal Processing, Vol. 38, No. 1, 2013, pp. 223-236. https://doi.org/10.1016/j.ymssp.2013.02.015

[11] Sree, D., and Stephens, D., "Improved Separation of Tone and Broadband Noise Components from Open Rotor Acoustic Data," Aerospace, Vol. 3, No. 3, 2016, p. 29.

[12] Antoni, J., Bonnardot, F., Raad, A., and Badaoui, M. E., "Cyclostationary Modelling of Rotating Machine Vibration Signals," Mechanical Systems and Signal Processing, Vol. 18, No. 6, 2004, pp. 1285-1314. https://doi.org/10.1016/S0888-3270(03)00088-8

[13] Javorskyj, I., Isayev, I., Majewski, J., and Yuzefovy, R., "Component Covariance Analysis for Periodically Correlated Random Processes," Signal Processing, Vol. 90, No. 4, 2010, pp. 1083-1102. https://doi.org/10.1016/j.sigpro.2009.07.031

[14] Braun, S., Mechanical Signature Analysis: Theory and Applications, 1st ed., Academic Press, New York, 1986, Chap. 9.

[15] Braun, S., "The Synchronous (Time Domain) Average Revisited," Mechanical Systems and Signal Processing, Vol. 25, No. 4, 2011, pp. 1087-1102. https://doi.org/10.1016/j.ymssp.2010.07.016

[16] Widrow, B., and Stearns, S. D., Adaptive Signal Processing, PrenticeHall, Upper Saddle River, NJ, 1985, Chap. 12.

[17] Nehorai, A., and Porat, B., "Adaptive Comb Filtering for Harmonic Signal Enhancement," IEEE Transactions on Acoustics, Speech, and Signal Processing, Vol. 34, No. 5, 1986, pp. 1124-1138.

[18] Parker, P. J., and Anderson, B. D., "Frequency Tracking of Nonsinusoidal Periodic Signals in Noise," Signal Processing, Vol. 20, No. 2, 
1990, pp. $127-152$. https://doi.org/10.1016/0165-1684(90)90124-H

[19] James, B., Anderson, B. D. O., and Williamson, R. C., "Conditional Mean and Maximum Likelihood Approaches to Multiharmonic Frequency Estimation," IEEE Transactions on Signal Processing, Vol. 42, No. 6, 1994, pp. 1366-1375.

[20] Truong, A., and Papamoschou, D., "Harmonic and Broadband Separation of Noise from a Small Ducted Fan," AIAA Paper 2015-3282, 2015. https://doi.org/10.2514/6.2015-3282

[21] Stephens, D. B., and Vold, H., "Order Tracking Signal Processing for Open Rotor Acoustics," Journal of Sound and Vibration, Vol. 333, No. 16, 2014, pp. 3818-3830. https://doi.org/10.1016/j.jsv.2014.04.005

[22] Shah, P. N., Vold, H., Hensley, D., Envia, E., and Stephens, D., "A HighResolution Continuous-Scan Acoustic Measurement Method for Turbofan Engine Applications," Journal of Turbomachinery, Vol. 137, No. 12, 2015, Paper 121002.

[23] Shah, P. N., White, A., Hensley, D., Papamoschou, D., and Vold, H., "Continuous-Scan Phased Array Measurement Methods for Turbofan Engine Acoustic Testing," Journal of Engineering for Gas Turbines and Power, Vol. 141, No. 8, 2019, Paper 081201.

[24] Assoumane, A., Sekko, E., Antoni, J., and Ravier, P., "Bearing Signal Enhancement Using Taylor- $H_{\infty}$ Estimator Under Variable Speed Condition," IEEE Transactions on Instrumentation and Measurement, Vol. 67, No. 11, 2018, pp. 2538-2547. https://doi.org/10.1109/TIM.2018.2828738

[25] Antoni, J., Leclère, Q., and Julliard, E., "Extraction des Composantes Aéroacoustiques Tonales et Larges Bandes," 14ème Congrès Français d'Acoustique: CFA 2018, 2018 (in French).

[26] Borghesani, P., Pennacchi, P., Chatterton, S., and Ricci, R., "The Velocity Synchronous Discrete Fourier Transform for Order Tracking in the Field of Rotating Machinery," Mechanical Systems and Signal Processing, Vol. 44, No. 1, 2014, pp. 118-133. https://doi.org/10.1016/j.ymssp.2013.03.026

[27] Capdessus, C., Sekko, E., and Antoni, J., "Speed Transform, a New Time-Varying Frequency Analysis Technique," Advances in Condition Monitoring of Machinery in Non-Stationary Operations, edited by G. Dalpiaz, R. Rubini, G. D'Elia, M. Cocconcelli, F. Chaari, R. Zimroz, W. Bartelmus, and M. Haddar, Springer, Berlin, 2014, pp. 23-35.

[28] Antoni, J., Abboud, D., and Baudin, S., "Time-Angle Periodically Correlated Processes," Cyclostationarity: Theory and Methods, edited by F. Chaari, J. Leśkow, A. Napolitano, and A. Sanchez-Ramirez, Lecture Notes in Mechanical Engineering, Springer Nature, Switzerland, AG, 2014, pp. 3-14.

https://doi.org/10.1007/978-3-319-04187-2

[29] Elie, B., and Chardon, G., "Robust Tonal and Noise Separation in Presence of Colored Noise, and Application to Voiced Fricatives," 22nd International Congress on Acoustics (ICA), 2016, https://hal .archives-ouvertes.fr/hal-01372313.

[30] Welch, P., "The Use of Fast Fourier Transform for the Estimation of Power Spectra: A Method Based on Time Averaging over Short, Modified Periodograms," IEEE Transactions on Audio and Electroacoustics, Vol. 15, No. 2, 1967, pp. 70-73. https://doi.org/10.1109/TAU.1967.1161901

[31] Magnus, J. R., and Neudecker, H., Matrix Differential Calculus with Applications in Statistics and Econometrics, 2nd ed., Wiley, New York, 1999, Chap. 9.

[32] Horváth, C., "Beamforming Investigation of Dominant Counter-Rotating Open Rotor Tonal and Broadband Noise Sources," AIAA Journal, Vol. 53, No. 6, 2015, pp. 1602-1611. https://doi.org/10.2514/1.J053465

[33] Tokaji, K., Soós, B., and Horváth, C., "Beamforming Method for Extracting the Broadband Noise Sources of Counter-Rotating Open Rotors," AIAA Journal, Vol. 58, No. 7, 2020, pp. 3028-3039. https://doi.org/10.2514/1.J058934

[34] Holste, F., and Neise, W., "Noise Source Identification in a Propfan Model by Means of Acoustical Near Field Measurements," Journal of Sound and Vibration, Vol. 203, No. 4, 1997, pp. 641-665. https://doi.org/10.1006/jsvi.1996.0890

[35] Lengani, D., Santner, C., Spataro, R., and Göttlich, E., "Analysis Tools for the Unsteady Interactions in a Counter-Rotating Two-Spool Turbine Rig," Experimental Thermal and Fluid Science, Vol. 42, Oct. 2012, pp. 248-257. https://doi.org/10.1016/i.expthermflusci.2012.05.010

[36] Enghardt, L., Bahrs, V., and Tapken, U., "Advanced Analysis of Tonal Noise from Asynchronous Counter-Rotating Fans by Means of a Low Sensor Count," AIAA Paper 2019-2549, 2019. https://doi.org/10.2514/6.2019-2549

[37] Brillinger, D. R., Time Series: Data Analysis and Theory, Soc. for Industrial and Applied Mathematics, New York, 1975, Chap. 4.

D. Papamoschou Associate Editor 